\title{
Diversity of Selaginella across altitudinal gradient of the tropical region
}

\author{
AHMAD DWI SETYAWAN ${ }^{1,2, \vartheta}$, JATNA SUPRIATNA ${ }^{1}$, DEDY DARNAEDI ${ }^{3}$, ROKHMATULOH $^{4}$, SUTARNO $^{2}$, \\ SUGIYARTO ${ }^{2}$ \\ ${ }^{1}$ Program of Conservation Biology, Department of Biology, Faculty of Mathematics and Natural Sciences, University of Indonesia, Depok 16424, West \\ Java, Indonesia \\ ${ }^{2}$ Department of Biology, Faculty of Mathematics and Natural Sciences, Sebelas Maret University. J1. Ir. Sutami 36A Surakarta 57126, Central Java, \\ Indonesia. Phone/Fax. +62-271-663375, `email: volatileoils@gmail.com \\ ${ }^{3}$ Division of Botany, Research Center for Biology, Indonesian Institute of Sciences, Cibinong-Bogor 16911, Indonesia \\ ${ }^{4}$ Department of Geography, Faculty of Mathematics and Natural Sciences, University of Indonesia, Depok 16424, West Java, Indonesia
}

Manuscript received: 17 November 2015. Revision accepted: 28 April 2016.

\begin{abstract}
Setyawan AD, Supriatna J, Darnaedi D, Rokhmatuloh, Sutarno, Sugiyarto. 2016. Diversity of Selaginella across altitudinal gradient of the tropical region. Biodiversitas 17: 384-400. Selaginella prefers moist environment for its growth and requires water for fertilization; therefore it is often found flourish in plateau and hilly mountains with high rainfall. The aim of this research was to know species diversity of Selaginella along altitudinal gradient in the southern part of Java, Indonesia. The research was conducted between July 2007 and January 2014across the altitudinal region of Java, Indonesia, which includes the districts of Wonosobo, Purworejo, Kebumen, Banjarnegara and its border areas, with altitudes between 0 and $2500 \mathrm{~m}$. asl. The research found 12 selaginellas species, 10 species collected from field research, namely: S. aristata, S. ciliaris, S. intermedia, S. involvens, S. opaca, S. ornata, S. plana, S. repanda, S. remotifolia, and $S$. uncinata; and two species only known from herbarium collection of Herbarium Bogoriense (BO), namely S. singalanensis and S. willdenowii. This research was the first publication of the Selaginella flora along altitudinal gradient from the coastal area to the high mountains of Central Java, Indonesia.
\end{abstract}

Keywords: diversity, Central Java, Selaginella, species, taxonomy

\section{INTRODUCTION}

Selaginellaceae Reinch. is a lycophyte family with only one genus namely Selaginella P. Beauv. (club moss, spikemoss). There are about 700-750 species of Selaginella in the world, which distributed cosmopolitan and pantropical in the tropics and sub-tropics regions (Tyron and Tyron 1982; Jermy 1990). In the islands of Southeast Asia (Malay Archipelago, Nusantara), there are more than 200 species of Selaginella (Camus 1997; Hassler and Swale 2002). Meanwhile, in Java, there are 25 species of Selaginella (Setyawan 2008). Selaginella is a difficult genus to be classified. A large number of Selaginella species are morphologically polymorphic and have high morphological similarity among them (Setyawan et al. 2012). This confusion led to almost every species having more than one name, such as S. ornata and S. involvens, which have high morphological variation and with more than 25 synonyms each (Hassler and Swale 2002).

Selaginella is a plant genus that prefers moist habitat and need water for fertilization. The southern part of Central Java has a diverse ecosystem which dominated by hilly area, ranging from the coastal areas to the middle and high mountains. Mountainous region with humid climate and abundant water sources throughout the year is a hotspot for its diversity. This species is likely to be the object of global climate change. Selaginella is widely distributed in shaded and moist places, although the distribution of each species can be limited depending on location and seasons. According to Setyawan et al. (2012), there were 10 species of Selaginella distributed from the coastal area to the montane rain forest in the southern part of Central Java. S. plana and S. ciliaris are important species for the lowland area, while $S$. remotifolia and $S$. opaca are important species for the highlands. Meanwhile, $S$. ornata and $S$. aristata are important species for the ecotone area. The presence of other species such as $S$. involvens, S. intermedia, S. repanda and S. uncinata are very limited and the distribution can not certainty be known. S. uncinata is an invasive species, presumably naturalized from ornamental plants. Setyawan et al. (2013) stated that the altitude of 1500 may be the upper limit for the distribution of $S$. aristata, S. ciliaris, S. involvens, $S$. ornata, S. plana, S. singalanensis, and S. zollingeriana in Java. Meanwhile, the altitude of $2100 \mathrm{~m}$ is probably the upper limit for the distribution of $S$. opaca and $S$. remotifolia.

Selaginella is useful for traditional medicine, especially for treating wounds, postpartum, menstrual disorders and body fit improvement (tonics). Biomedical research shows that this plant has potential as an anti-oxidant, antiinflammatory, anti-cancer, antibacterial, etc (Setyawan 2011). Selaginella is also useful as an ornamental plant, handicraft materials, and vegetables (Winter and Jansen 2003; Setyawan 2009). Selaginella has a very diverse phytochemical content, such as: alkaloids, phenols (flavonoids, tannins, saponins), and terpenoids (triterpene, steroid) (Setyawan 2011; Chikmawati et al. 2012). These herbs also contain lignin, lignans, lignanosides, alkaloids, selaginellin, glycosides, glucosides, glycosyl flavones, and 
others. Some species contain certain natural products, such as ecdysteroid, essential oils and trehalose (Setyawan 2011).

Research on the flora of Selaginella along altitudinal gradient in the southern Central Java, Indonesia has never been done. However, it has been previously reported the altitudinal distribution of Selaginella in this region (Setyawan 2012). Meanwhile, previous research on the taxonomy of Selaginella in all over Java by Alston (1934a) has not been updated. This research aims to determine the flora of Selaginella along altitudinal gradient in the southern part of Central Java, Indonesia.

\section{MATERIALS AND METHODS}

\section{Study areas}

This research was conducted along altitudinal gradient in southern Central Java, Indonesia, known as historic area of Bagelen and surrounding areas. Administratively, the studied area mainly includes Wonosobo, Purworejo, Kebumen and Banjarnegara districts; since distribution of wildlife can not be restricted by administrative boundaries, the border region of neighboring district is also observed, i.e. part of Temanggung, Magelang, Kulonprogo, Banyumas, Purbalingga, and Pekalongan districts. All districts belong to Central Java Province, except for Kulonprogo that belongs to Yogyakarta Special Region (Figure 1). Geographically, this area is located between $-7.1046^{\circ}$ and $7.9033^{\circ}(\mathrm{S})$ and between $109.3341^{\circ}$ and $110.2298^{\circ}(\mathrm{E})$, the total area of more than $4200 \mathrm{~km}^{2}$, with a population of more than 4.5 million people. The altitude varies from $0 \mathrm{~m}$ in the southern coastal area to $3371 \mathrm{~m}$. asl. at the top of Mt. Sumbing; the temperature is ranging between $24-34^{\circ} \mathrm{C}$, but in the Dieng Plateau and surrounding area it ranged from $12-20^{\circ} \mathrm{C}$; relative humidity is $70-94 \%$, the average rainfall in the southern part is around $2300 \mathrm{~mm}$, and in the northern hilly part is more than $4200 \mathrm{~mm}$, with the highest rainfall is in the Garung sub-district, Wonosobo approximately 4802 mm (BPS Banjarnegara 2011, BPS Kebumen 2011, BPS Purworejo 2011, BPS Wonosobo 2011). This area has only two dry months in the north and 5-6 dry months in the south (Oldeman 1975). The main physiographic of this region are hilly mountains in the north consist of Dieng Plateau, highland of Mts. Sindoro and Sumbing, lowland area in the south along the coastal line, and Serayu Watersheds and South Serayu Mountains in the center. Soil types in the Dieng Plateau and southern lowlands are alluvial, the other highland areas are dominated by regosol or andosol soils, the southern lower mountains are dominated by latosol-andosol, complex-podzolic-latosol lithosol or regosol soils, whereas the karst region of Karangbolong has a complex mediterranian-regosolgrumosol soil (SRI 1960). More than half of the region's land is used for dry land farming, about a quarter is used for wet rice fields, the remainder is state forest, private plantations, rivers, lakes, developing areas (settlements, roads), etc (BPS Banjarnegara 2011, BPS Kebumen 2011, BPS Purworejo 2011, BPS Wonosobo 2011).Outside the forest, wild species inhabit abandoned places, such as river banks, edges of roads and irrigation canal, and fill in the sidelines of agricultural land, residential, grave and other built areas (Setyawan et al. 2012).

The field work was carried out of more than six years, between July 2007 and January 2014. Several massive surveys of Selaginella had been conducted, with altitude between 0 and $2500 \mathrm{~m}$. asl., both in wet and dry seasons. In this study, most of the studied area has been developed, viz. paddy and dry fields, agroforestry (community forests), plantation and production forest, home garden, settlements, roads, water bodies and drainages, etc. Natural forest is only found in a limited size in the slope of Dieng Mountains, Mt. Sindoro and Mt. Sumbing, which periodically burned. This research is mainly conducted in shaded and humid places, especially in the hilly areas of Wonosobo which have higher annual rainfall $(>4000 \mathrm{~mm}$ in 2011), one of the rainiest district in Java, but also along drier area in the southern coastal area.

\section{Procedures}

Almost all sites in the southern Central Java are anthropogenic area and influenced by human activities. Selaginella is generally found in abandoned places that are moist and shady, such as roadside cliffs, footpaths, tributaries cliffs, moist cliffs of forest, agroforests, and agricultural lands, etc. Some species can also grow in relatively open sites, such as pine forest (Pinus merkusii), the settlements and agricultural land. Selaginella rarely grows under a dense clump of herbs or shrubs; that place does not provide space and light for growth. All Selaginella species were recorded and collected as herbarium specimens and living collection for the experimental garden in Kejiwan, Wonosobo, Central Java (768 m. asl.). Both living plants and herbarium specimens were observed. Specimens of field collection were deposited at Herbarium Soloense (SO), Sebelas Maret University, Surakarta, Indonesia and some selected specimens were sent to Herbarium Bogoriense (BO), Indonesian Institute of Sciences, Cibinong-Bogor, Indonesia. A total of 440 herbarium specimens of Selaginella have been collected from the study site, and 18 historical herbarium specimens of BO from this area have also been observed. Since the existence of two species is only known from BO collections, the same plant species planted in the experimental garden is also observed, namely: $S$. singalanensis of Mt. Lawu, Central Java (ADS 467) and $S$. willdenowii of Mt Halimun Salak, West Java (ADS 460). Each herbarium specimen was unique, distinguished by location and time of collection. Data passport collected along with the specimens were used as standard for herbarium specimens. The specimens were identified by using several early literatures on Selaginella in Malay Archipelago, i.e. Alderwereld van Rosenburgh (1915a,b, 1916, 1917, 1918, 1920, 1922) and Alston (1934a, 1935a,b, 1937, 1940); and were compared with the specimens collection at BO, especially the specimens that had been determined by A.G.H. Alston before; and also by using several newest references from Malay Archipelago and adjacent area such as Wong $(1982,2010)$, Tsai and Shieh (1994), Li and Tan (2005), Chang et al. (2012), and Zhang 
et al. (2013), as well as previously study in Java such as Setyawan et al. (2012, 2013), Setyawan (2014), Setyawan (2015a,b), Setyawan et al. (2015a,b,c), and Setyawan and Sugiyarto (2015).

In addition to direct observations, the literatures were used to guide the preparation of description. Meanwhile, the synonyms and global distribution was mainly according to Hassler and Swale (2002) and Chang et al. (2012). Locality is according to the administrative division of subdistrict level.

\section{RESULTS AND DISCUSSION}

\section{Description}

In this study, a total of 13 species of Selaginella have been identified along altitudinal gradient of the southern of Central Java, Indonesia. The 11 species was found from the field surveys, namely $S$. aristata, $S$. ciliaris, $S$. intermedia, S. involvens, S. opaca, S. ornata, S. plana, S. remotifolia, $S$. repanda, and $S$. uncinata. While, the two species were only found from the historic collection of Herbarium Bogoriense specimens, namely $S$. singalanensis and $S$. willdenowii. The altitudinal distribution of each species is showed in Figure 2A-L.

Selaginella is a rarely annual (S. aristata, $S$. ciliaris) or perennial herb; terrestrial, epiphythic ( $S$. involvens, $S$. repanda), or occasionally epiphytic (S. ciliaris). Stems are leafy, slender, descending ( $S$. aristata), creeping and rooting at intervals ( $S$. ciliaris, $S$. opaca, $S$. remotifolia, $S$. singalanensis), ascending ( $S$. aristata, S. plana), decumbent (S. repanda) or erect, roll up when dry (S. involvens), with or without branches on lower part, rooting near base, branching dichotomously, regularly or irregularly branched. Rhizomes rarely present ( $S$. involvens, $S$. repanda).

Rhizophores are present or absent (S. involvens), geotropic, borne on dorsal (upper) or ventral (lower) side in axils of branches, throughout (creeping ones), or confined to base $(S$. ornata). Roots formed at tip of rhizophore, branched. Leaves are small, simple, with a single vein (exceptionally veins forked), always bearing an inconspicuous ligules in axil on adaxial side (only prominent in early development), monomorphic or dimorphic; vegetative leaves (tropophyll) are monomorphic-spirally arranged at basal main stem and dimorphic-4 lanes arranged on other parts ( $S$. involvens, $S$. plana), or in most species dimorphic and usually arranged in 2 median (ventral) or upper side and 2 lateral (dorsal) or lower side rows on all branches $(S$. ornata, $S$. singalanensis); median leaves are usually smaller, and in different shape from the lateral leaves; axillary leaves are single borne at the forking of each branch, being somewhat different from other leaves. Strobilus (clusters of imbricating sporophylls) are usually terminal on the ends

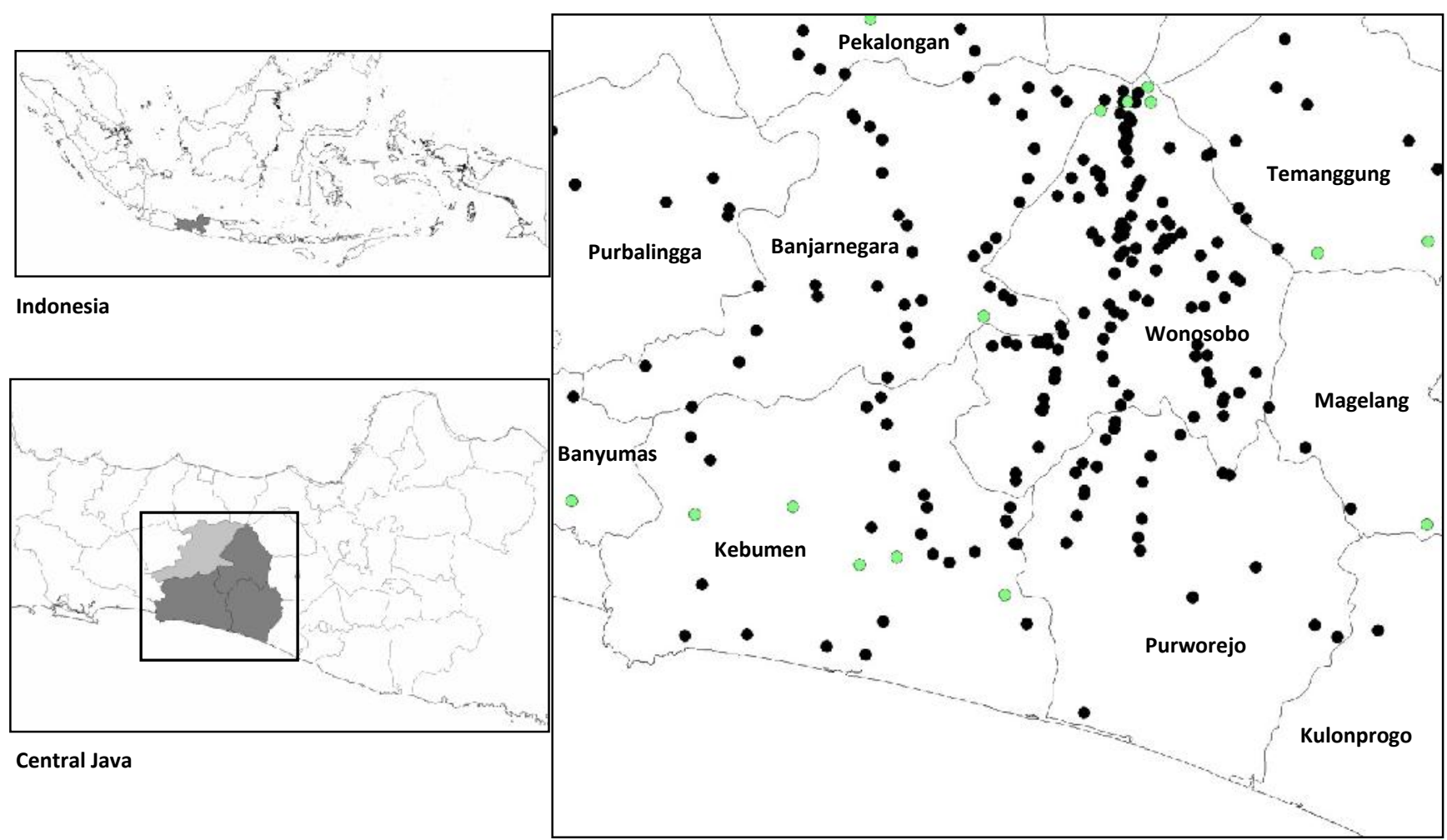

Southern Central Java

Figure 1. Study sites of selaginellas altitudinal diversity in the southern Central Java, Indonesia, namely: (i) Herbarium specimens from field collection (O), and (ii). Herbarium specimens from Herbarium Bogoriense (BO) (O) 

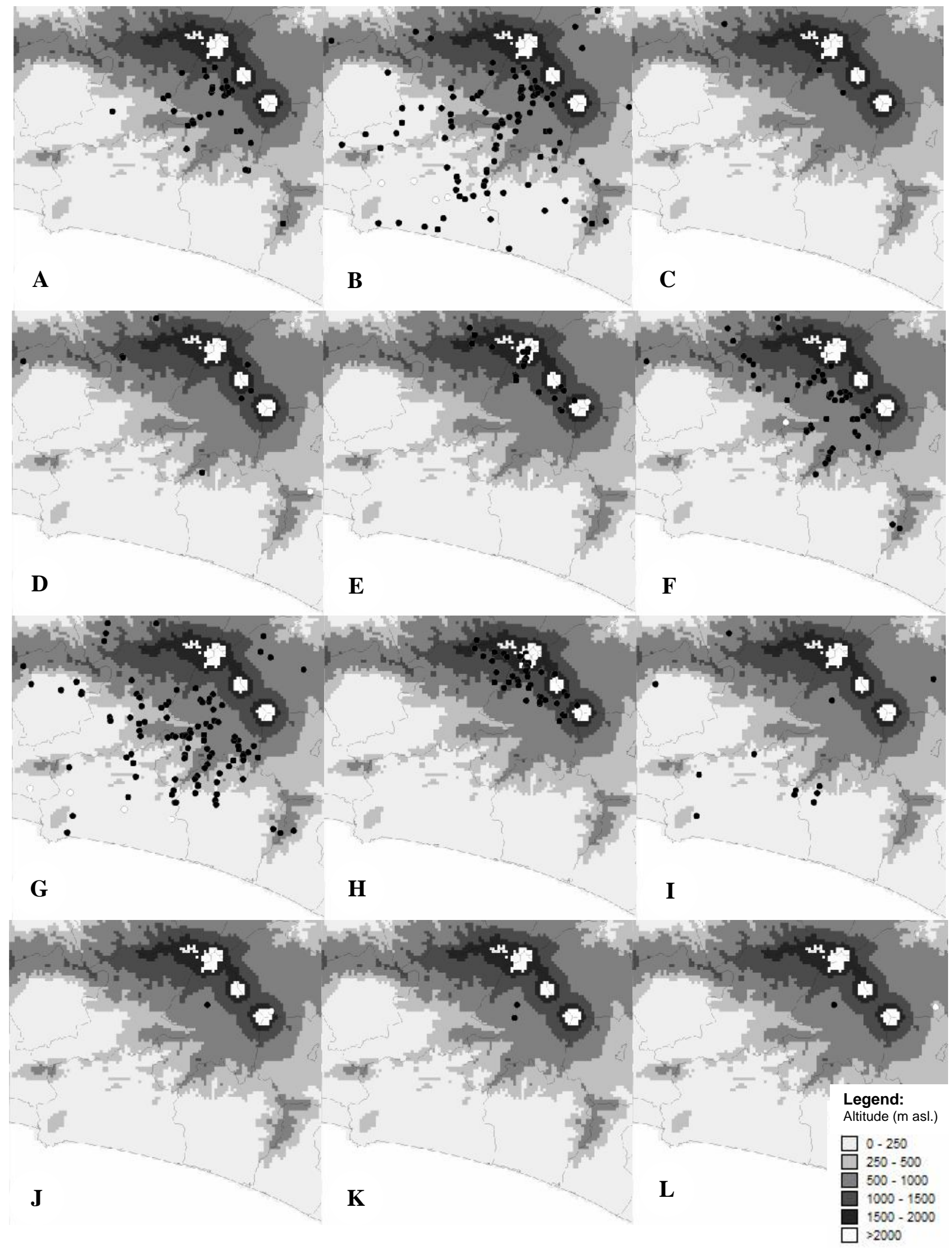

Figure 2. Species distribution of selaginellas in Southern Java and the surrounding; A. S. aristata, B. S. ciliaris, C. S. intermedia, D. S. involvens, E. S. opaca, F. S. ornata, G. S. plana, H. S. remotifolia, I. S. repanda, J. S. singalanensis, K. S. uncinata, and L. S. willdenowii 
and sides of branches, cylindrical, tetragonal (S. involvens, $S$. opaca, S. remotifolia), flattened (S. ciliaris) or do not in compact strobilus ( $S$. aristata, sometimes). Sporophylls (fertile leaves) are monomorphic or adjacently different, slightly or highly differentiated from vegetative leaves. Sporangia are short-stalked, solitary in an axil of sporophylls, opening by distal slits. Spores are of two types (heterosporous), megaspores tetrad (1-2-)4, large, commonly at the base of strobilus, microspores numerous (hundreds), minute; sporangia round or oval, opening by a transverse slit. White, black, brown, or reddish to yellowish red $(S$. repanda).

One genus and ca. 700-750 species: almost cosmopolitan, with its highest diversity in the tropics; 200 species in Malay Archipelago, 25 (5 endemic) species in Java, 13 species in southern Central Java.

\section{Key to species}

1. Stem (sub-)erect, rooting at base, or bearing rhizophores

2. Stem shorter than $50 \mathrm{~cm}$

3. Stem fleshy ..................................... S. aristata

3. Stem fragile ........................................ S. ornata

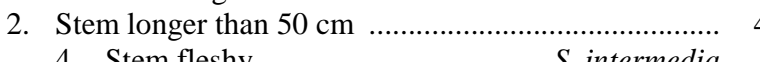

4. Stem fleshy .................................... S. intermedia

4. Not so

5. Stem hard, caulescent, easily broken ............. .................................... S. involvens

5. Stem tough ….............................. S. plana

1. Stem mostly creeping, rooting at intervals .......................... 6

6. Leaves with blue iridescence ................................... 7

6. Not so

7. Stem creeping, shorter than $1 \mathrm{~m}$........ S. uncinata

7. Stem creeping to scrambling (viselike), longer than $1 \mathrm{~m}$.................................... S. willdenowii 8. Stem shorter than $15 \mathrm{~cm}$............... S. ciliaris

8. Stem more than $15 \mathrm{~cm}$...............................

9. Stem fleshy .............................. S. opaca

9. Not so

10. Leaves loosely arranged ....................

.................................. S. remotifolia

10. Leaves imbricate .............................. 11

11. Spores bright yellow...................

11. Not so ............... S. singalanensis

\section{Species description}

Selaginella aristata Spring, Bull. Acad. Brux. 10: 232, no. 152 (1843) (Figure 3.A)

Synonym: Lycopodioides leptophylla (Baker) Kuntze, Revis. Gen. Pl. 2: 826 (1891); Lycopodium philippense Willd. ex Spreng., Syst. Veg. 4: 17 (1827); Lycopodium remotifolium Desv., Prodr. Fil., Ann. Soc. L. Paris, 6: 190, no. 155 (1827); Selaginella aristata var. brevifolia Hieron., Elmer's Leaflets Philip. Bot. 6: 2045 (1913); Selaginella aristata var. kaudernii Alderw., Bull. Jard. Bot. Buit. III, 5: 238 (1922); Selaginella aristata var. obtusifolia Hieron., Elmer's Leaflets Philip. Bot. 6: 2046 (1913); Selaginella circinalis var. aristata Presl., Bot. Bem. 153 (1845); Selaginella cristata Warb., Monsunia I: 125
(1900);Selaginella hayata Satake, Bot. Mag. (Tokyo) 48 (568): 261 (1934);Selaginella leptophylla Baker var wichurae (Warb.) Tagawa, Acta Phytotax. Geobot. 10 (3): 194 (1941); Selaginella leptophylla Baker, J. Bot. 23: 157 (1885); Selaginella pickeringii Hieron., Elmer's Leaflets Phil. Bot. 6: 2046 (1913); Selaginella proniflora auct. non (Lam.) Baker, Matsum. \& Hayata, Enum. Pl. Formosa 554 (1906); Selaginella satakeana Koidz., Acta Phytotax. Geobot. 5: 40 (1936); Selaginella stenostachya Hayata, Icon. Pl. Formosan. 4: 129 (1914); Selaginella wichurae Warb., Monsunia I: 127 (1900).

Annual herb, small, fleshy, sub-erect, prostrate or ascending, caespitose, glabrous, fan-shaped, whitish green leaves with pink to brown stem, very fleshy; multiple branched at main stem, forming dendritic stem. Stems decumbent to ascending, multiple branched or dendritic, especially at the mature ones, ca. 4-21 cm long, 3-6 mm wide (including leaves). Rhizophores present at basal stem, originated from the ventral side of branching stem, ca. 1 $\mathrm{mm}$ in diam. Leaves (trophophylls) dimorphic, arranged in 4 lanes (2 lateral, 2 median), sparsely arranged at main stem but closely arranged at the branches, vein single; lateral (ventral) leaves lanceolate, oblong or ovate at main stem, lanceolate to falcate at branches, 2-3 mm long, 1-2 $\mathrm{mm}$ wide, apex acute or obtuse, asymmetrical, base subcordate or rounded, margin serrulate to subentire; median (dorsal) leaves smaller than the lateral ones, lanceolate to ovate, more or less symmetrically, $1.5-2 \mathrm{~mm}$ long, 0.5-1 mm wide, apex caudate to long tail-like, apices upward or bended back, base obtuse, margin serrulate, single vein reaching the apex; axillary leaves lanceolate, ovate or subcordate, 1.5-3 mm long, 0.5-1.5 mm wide, apex obtuse, base rounded, margin serrulate, single vein nearly reaching the apex. Strobilus solitary, terminal, loosely, bisymmetrical, upper-plane sporophylls longer than lowerplane, up to $1 \mathrm{~cm}$ long.

Habitat and ecology: It widely grows on moist steep cliffs; rugged contours allow plenty of water seepage, humid and suitable for its growth. It was found on tributary cliff, small shaded river bank, the cliff and edge of irrigation canals, the cliff of water springs, river bridge, the cliff and edge of main roads, villages roads, footpath; on the steep cliff of acacia, albizia, and bamboo stands, on the dry field, agroforestry, pine and agathis forests; on the palawija crops, salak and vegetable fields; on the intensive agricultural field shaded by paranet; on the village cliffs and cemetery hills. It was only abundant in the rainy season, at altitude of 186-1209 m. asl.

Locality: Banjarnegara (Bawang, Madukara, Pagentan, Sigaluh), Purworejo (Kaligesing), Wonosobo (Garung, Kaliwiro, Kepil, Kertek, Mojotengah, Sapuran, Selomerto, Wadaslintang, Wonosobo-city).

Distribution: China (Guangxi, Guizhou, Henan, Hongkong, Sichuan, Yunan), India, Indonesia, Japan, Myanmar, Philippines, Sri Langka, Taiwan, Thailand, Vietnam. In Indonesia: Java, Moluccas (Buru, Halmahera, Seram, Ternate), Sulawesi.

Specimens observed: ADS 190, ADS 196, ADS 201, ADS 203, ADS 204, ADS 207, ADS 212, ADS 219, ADS 235, ADS 240, ADS 244, ADS 247, ADS 254, ADS 285, 
ADS 301, ADS 307, ADS 309, ADS 317, ADS 332, ADS 343, ADS 344, ADS 351, ADS 353, ADS 358, ADS 364, ADS 367, ADS 370, ADS 396, ADS 398, ADS 405, ADS 410, ADS 521, ADS 534, ADS 537, ADS 541, ADS 554, ADS 740.

Note: It is a fleshy annual species that only abundant in the rainy season; cover-abundance in the rainy season can reach four times the dry season. In the dry season, it generally will only be remnants that led to death. This species generally co-habitation with $S$. ciliaris, especially in moist and shaded places, while in a very open place where $S$. ciliaris can still grow, $S$. aristata is unable to grow.

Selaginella ciliaris (Retz.) Spring, Bull. Acad. Brux. 10: 23 (1843) (Figure 3.B-C).

Basionym: Lycopodium ciliare Retz., Observ. Bot. 5: 32 (1789).

Synonym: Lycopodioides ciliaris (Retz.) Kuntze, Rev. Gen. Pl. 1: 826 (1891); Lycopodioides depressa (Sw.) Kuntze, Rev. Gen. Pl. 1: 826 (1891); Lycopodioides exigua (Spring) Kuntze, Rev. Gen. Pl. 1: 826 (1891); Lycopodioides pumilio (R. Br.) Kuntze, Rev. Gen. Pl. 1: 827 (1891); Lycopodium belangeri Bory, Bel. Voy. Bot. 2: 12 (1833 or 1834); Lycopodium depressum Sw., Schrad. Jour. Bot (1800) (2): 110. 1801; Lycopodium pumilio R. Br., Prodr. Fl. Nov. Holland 166 (1810); Selaginella belangeri (Bory) Spring, Monogr. Lyc. II: 242: 180 (1850); Selaginella belangeri f. olivacea Alderw., Mal. Fern Allies, 173 (1915); Selaginella congregata Alderw., Bull. Jard. Bot. Buit. III, 2: 179 (1920); Selaginella depressa (Sw.) Spring, Bull. Acad. Brux. 10: 234, no. 162 (1843); Selaginella exigua Spring, Mem. Acad. Sci. Belg. 24 (2): 238 (1850); Selaginella papana Alderw., Bull. Jard. Bot. Buit. III, 5: 235 (1922); Selaginella proniflora Baker, Jour. Bot. 22: 156, no. 260 (1885); Selaginella pumilio (R. Br.) Spring, Bull. Acad. Brux. 10: 232, no 143 (1843); Selaginella tenera sensu Narayanaswami \& Carter, Mem. As. Soc. Beng. 7: 290 (1922); Selaginella ujensis Hieron., Bot. Jahrb. Engl. 44: 514 (1910); Selaginella winkleri Hieron., Bot. Jahrb. Engl. 44: 516 (1910); Stachygynandrum ciliare (Gmel.) Beauv., Prodr. Aetheog. 110 (1805); Stachygynandrum depressum (A. Br.) Carr., Cat. Afr. Pl. coll. by Dr. F. Welwitsch in (1853)-1861, 2 (2): 262. 1901; Stachygynandrum depressum var. minus (A. Br.) Carr., Cat. Afr. Pl. coll. by Dr. F. Welwitsch in (1853)1861, 2 (2): 262. (1901).

Annual herb, small, shortly creeping, prostrate or ascending, sometimes fan-shaped, stramineous, $2-12.5 \mathrm{~cm}$ long, glabrous, angular or sulcate, rooting at intervals but mostly near base, sometimes in a clump, green, yellowish or dark brown. Stems decumbent, branched throughout without a significant main stem, 4-5 mm wide (including leaves). Rhizophores present at intervals but mostly near the base, originated from the ventral side in axils of branches, ca. $0.3 \mathrm{~mm}$ in diam. Leaves dimorphic, arranged in 4 lanes (2 lateral, 2 median), vein single; lateral leaves ovate to lanceolate, more or less symmetrical, $1.5-2 \mathrm{~mm}$ long, 0.6-1 $\mathrm{mm}$ wide, apex acuminate or acute, base rounded or subcordate, margin serrulate or ciliate, single vein reaching the apex, keeled, pointing outwards; median leaves ovate to falcate, contiguous, nearly asymmetrical, 2$2.5 \mathrm{~mm}$ long, 0.6-1.5 mm wide, apex acute, aristate or cuspidate, base subcordate or rounded, margin serrulate but laciniate at basal part, pointing upwards, minutely toothed, ciliate, slightly carinate, midrib prominent, single vein reaching or nearly reaching the apex; axillary leaves lanceolate to ovate, equally sided (bisymmetrically) or slightly asymmetrical,1.5-2.5 mm long, 1-1.5 mm wide, single vein reaching or nearly reaching the apex, apex acute, base rounded to subcordate, exauriculate, ciliate, margin ciliate or laciniate at basal part and finely toothed or serrulate at apical part. Strobilus are solitary (or twin), terminal, compact, dorsiventrally complanate or flattened, up to ca. $0.5-2 \mathrm{~cm}$ long; sporophylls strongly dimorphic, spore greenish-yellowish orange.

Habitat and ecology: It is strongly influenced by growth season. Since it has small size and short roots, the plants die during the dry season and sprout new plantlets at the beginning of the rainy season (r-selection). Its habitat is the moist rocky slope, slightly open and shady places without excessive soil or humus, but it is sometimes also found on steep soil and flat ground rather open and moist. It is found in a variety of building walls and natural rocky cliffs, such as near water spring, small river, large and small irrigation canals, roadside ditch, fences and houses, ruins of abandoned housing, fish pond, tower, river bridge, cemetery and tombstones, terassering of fields, and roadside sandstones; around the footpath, dirt road, village road, main road, rice field dikes, fields of palawija, salak, cassava and vegetables, tea gardens, coffee plantations, agroforestry of albizia, forest of pine and agathis; intensive agricultural fields shaded by paranet; and the base of palm trees in the coastal areas. It is very often found in the cemetery. The locations with shaded large trees and deprived herbs and shrubs provide enough space for growth without significant competition. Setyawan (2011) found that this species is used as a tomb cover. It is found at altitudes of 11-1246 m. asl.

Locality: Banjarnegara (Banjarmangu, Banjarnegaracity, Bawang, Madukara, Mandiraja, Pagedongan, Pagentan, Pejawaran, Purwanegara, Sigaluh, Wanadadi), Banyumas (Somagede), Kebumen (Buayan, Buluspesantren, Karangsambung, Klirong, Mirit, Petanahan, Prembun, Gombong, Karanganyar, Kebumen-city), Kulonprogo (Girimulyo), Magelang (Salaman, Secang), Pekalongan (Paninggaran), Purbalingga (Kejobong, Karangmoncol), Purworejo (Bayan, Bener, Grabag, Kaligesing, Loano), Temanggung (Bejen, Jumo), Wonosobo (Garung, Kaliwiro, Kertek, Mojotengah, Selomerto, Wadaslintang, Wonosobo-city).

Distribution: N-Australia, S-China (Guangdong), India, Indonesia, Marianas, Micronesia, Myanmar, New Guinea, Palau Isl., Philippines, Sri Lanka, Solomons Isl., Taiwan, Thailand, Vietnam. In Indonesia: Java, Sulawesi, Moluccas (Ternate).

Specimens observed: ADS 78, ADS 91, ADS 176, ADS 182, ADS 191, ADS 194, ADS 200, ADS 202, ADS 205, ADS 206, ADS 211, ADS 213, ADS 215, ADS 218, ADS 236, ADS 239, ADS 243, ADS 248, ADS 250, ADS 
255, ADS 258, ADS 284, ADS 302, ADS 306, ADS 310, ADS 314, ADS 316, ADS 319, ADS 320, ADS 324, ADS 327, ADS 331, ADS 333, ADS 342, ADS 345, ADS 349, ADS 354, ADS 359, ADS 363, ADS 366, ADS 369, ADS 372, ADS 375, ADS 376, ADS 395, ADS 401, ADS 404, ADS 407, ADS 409, ADS 414, ADS 433, ADS 445, ADS 516, ADS 522, ADS 524, ADS 525, ADS 526, ADS 527, ADS 528, ADS 529, ADS 530, ADS 531, ADS 532, ADS 533, ADS 540, ADS 549, ADS 574, ADS 616, ADS 617, ADS 618, ADS 619, ADS 620, ADS 621, ADS 622, ADS 623, ADS 734, ADS 738, ADS 742, ADS 743, ADS 744, ADS 1055, ADS 1059, ADS 1061, ADS 1063, ADS 1065 , ADS 1070, ADS 1073, ADS 1074, ADS 1075, ADS 1076, ADS 1077, ADS 1083, ADS 1084, ADS 1085, ADS 1087, ADS 1098, ADS 1100, ADS 1129, ADS 1131, ADS 1134, ADS 1142, ADS 1405, ADS 1421, ADS 1425, ADS 1426, ADS 1428, ADS 1452, ADS 1456, ADS 1460, ADS 1463, Banjoemas 2219 (BO!), Banjoemas 2369 (BO!), Banjoemas 2436 (BO!), Banjoemas 2849 (BO!), R. Brinkman 75 (BO!), as well as ADS 303, ADS 311, ADS 346, ADS 350, ADS 360, ADS 446, ADS 1404, ADS 1406 , and ADS 1419 that have broadly ovate to oblong leaves similar to Selaginella devolii H.M. Chang, P.F. Lu \& W.L. Chiou, Blumea 56 (1): 21 (2011)

Note: It has a very diverse morphological characteristic. At the same place, two individuals can grow very different in shape, size and color; a brief observation will regard it as a distinct species. Figure 3.B shows two individuals of $S$. ciliaris growing side by side, but has a much different color, i.e. bright green and dark brown. Andrews (1990) has provided a clear description of some morphological variation of Selaginella ciliaris complex, which can be guided so as not separating this species without a clear and definite concept. It is also found a variant of $S$. ciliaris that have smaller size, more rounded leaves shape and tend to grow solitary, in the highland, that similar to $S$. devolii.

Selaginella intermedia (Blume) Spring, Bull. Acad. Brux. 10: 144 (1843) (Figure 3.D).

Synonym: Lycopodioides atroviridis (Wall.) Kuntze, Rev. Gen. Pl. 1: 825 (1891); Lycopodioides plumea (Spring) Kuntze, Rev. Gen. Pl. 1: 827 (1891); Lycopodium atroviride Hook. \& Grev., Ic. Fil. 1: t. 39 (1831); Lycopodium atro-viride Wall., Cat., 6, no. 120 (1829); Lycopodium cuspidatum Hook., ms.; Lycopodium furcatum Roxb. ex Griff., Calc. Jour. Nat. Hist. 4: 475 (1844); Lycopodium furcatum Roxb. in Wall., Cat. 62, no. 120 (1829) [nomen]; Lycopodium hymenophyllum Roxb., ms.; Lycopodium intermedium Blume, Enum. Pl. Jav. 2: 269, no. 20 (1830); Sclaginellabimarginata Alderw., Bull. Jard. Bot. Buit. III, 5: 231 (1922); Selaginella ascendens Alderw., Bull. Jard. Bot. Buit. II, 11: 33 (1913); Selaginella atrovirens (Wall.) Spring, Flora, 21: 183 (1838); Selaginella caudata sensu F. von Muell., Descr. Notes Papuan PI. 1 (4): 75 (1876); Selaginella consobrina Alderw., Bull. Jard. Bot. Buit. III, 2: 178 (1920); Selaginella cuprea Ridl., Jour. Roy. As. Soc. Str. Br. 80: 152, no. 14 (1919); Selaginella cuprea var. major Ridl., Jour. Roy. Asiatic Soc., Str. Br., 80: 152 (1919); Selaginella pentaphlebia Alderw., Bull. Jard. Bot. Buit. III,
5: 228 (1922); Selaginella plumea f. typica Alderw., Mal. Fern Allies 102 (1915); Selaginella plumea Spring, Monogr. Lyc. II: 186 (136), no. 81 (1850); Selaginella pseudovenulosa Alderw., Bull. Jard. Bot. Buit. III, 5: 230 (1922); Selaginella sibogana Alderw., Bull. Jard. Bot. Buit. II, 11: 30 (1913); Selaginella sibogana f. typica Alderw., Bull. Jard. Bot. Buit. II, 16: 44 (1914); Selaginella sibogana var. subbinervia Alderw., Bull. Jard. Bot. Buit. II, 16: 44 (1914); Selaginella similis Kuhn, Forschungsr. Gazelle 4 (Bot.): 17 (1889); Selaginella spurie-marginata Alderw., Bull. Jard. Bot. Buit. III, 5: 231 (1922); Selaginella trinervia Spring, Bull. Acad. Brux. 10: 143, no. 62 (1843); Selaginella vanvuureni Alderw., Bull. Jard. Bot. Buit. II, 16: 44 (1914); Selaginella venulosa Alderw., Bull. Jard. Bot. Buit. II, 28: 47 (1918); Selaginella wigmanii Alderw., Bull. Jard. Bot. Buit. II, 11: 32 (1913); var. dolichocentrus K.M.Wong, Gard. Bull. Singapore 35 (2): 125 (1982) publ. 1983.

Perennial herb, suberect to ascending, glabrous, membranous, multiple branched at main stem, up to ca 70 $\mathrm{cm}$ long, pale green leaves. Stems suberect to ascending, cylindrical, glabrous, multiple branched toward apex, 3-5 $\mathrm{mm}$ wide (including leaves). Rhizophores present at basal stem, thick, cylindrical, originated from the ventral side of branching stem, ca. 1-1.5 $\mathrm{mm}$ in diam. Leaves dimorphic, arranged in 4 lanes (2 lateral, 2 median), sparsely arranged at main stem but closely arranged at the branches, vein single; lateral leaves oblong or ovate at main stem, lanceolate at branches, 4-5 mm long, 1.5-2.5 $\mathrm{mm}$ wide, apex acute or acuminate, asymmetrical, base rounded, margin dentate; median leaves smaller than the lateral ones, obovate, oblique at base, more or less symmetrically, 3-4 $\mathrm{mm}$ long, 1-2 $\mathrm{mm}$ wide, apex aristate, base rounded or cordate, margin dentate; axillary leaves lanceolate or ovate, 2-3 mm long, 1.5-2 mm wide, apex acute or obtuse, base rounded, margin dentate-denticulate, single vein nearly reaching the apex. Strobilus solitary, terminal, loosely, quadrangular, up to $5 \mathrm{~cm}$ long; sporophylls monomorphic.

Locality: Wonosobo (Mojotengah, Wonosobo-city)

Habitat and ecology: It was found on the steep cliff of small river banks and river banks shaded by lush of bamboo; at altitude of $1012-1131 \mathrm{~m}$. asl.

Distribution: India (Kerala, Tamil Nadu), Indonesia, Malaysia (Peninsular, Mt. Kinabalu), Myanmar, Sri Lanka, Thailand, Vietnam,. In Indonesia: Java, Sumatra, Sulawesi.

Specimens observed: ADS 438, ADS 441, ADS 552, ADS 1104.

Note: This species is typical of the western Java highlands, and it is very rare in Central Java. In this study, it was only found two specimens in the southern slopes of Dieng mountains. In observation of BO collection, only one specimen that can be confirmed as S. intermedia of Central Java, i.e. from the northern slope of the Dieng mountain (Doro, Pekalongan; Docters v. Leeuwen 453, 7 June 1912). Not far from that place, it was also found this species (Paningaran, Pekalongan; ADS 1104, 28 August 2013). 
Selaginella involvens (Sw.) Spring, Bull. Acad. Brux. 10: 136, no. 6 (1843) (Figure 3.E)

Basionym: Lycopodioides involvens (Sw.) Kuntze, Rev. Gen. Pl. 1: 826 (1891).

Synonym: Lycopodioides pennula (Desv.) Kuntze, Rev. Gen. Pl. 1: 827 (1891); Lycopodium caulescens Wallex Hook. \& Grev., Enum. Fil. Hook. Bot. Misc. 2: 382 (1831); Lycopodium circinale auct. non L., Thunb., Fl. Jap. (Thunberg) 341 (1784); Lycopodium involvens Sw., Syn. Fil., 182: 50 (1806); Lycopodium microstachyum Desv. in Poir., Lamarck, Encycl. Suppl. 3: 554, no 97. [1813] 1814; Selaginella bellula Cesati, Atti Acad. Napoli, 7 (8): 36 (1876); Selaginella caudispica Alderw., Bull. Jard. Bot. Buit. II, 11: 35 (1913); Selaginella caulescens (Wall. ex Hook. \& Grev.) Spring, Bull. Acad. Brux. 10 (1): 137 (1843); Selaginella caulescens f. minor Hieron., Bot. Tidsskr. 24: 114 (1901) [nomen; Selaginella caulescens f. typica Alderw., Mal. Fern Allies, 138 (1915); Selaginella caulescens var. belulla (Ces.) Hieron., Hedwigia 50: 4 (1910); Selaginella caulescens var. brachypoda Baker, Jour. Bot. 23: 24 (1885); Selaginella caulescens var. gracilis Bull., Cat. 164: 7 (1880); Selaginella caulescens var. japonica (Moore ex McNab) Baker, Jour. Bot. 23: 24 (1885); Selaginella caulescens var. minor Milde, Fil. Eur., 270 (1867); Selaginella caulescens var. minor Sandford, Man. Exot. Ferns \& Selag., 258 (1882); Selaginella caulescens var. subintegerrima Spring, Monogr. Lyc. II: 159 (1850); Selaginella homomorpha Klotzsch ex Milde, Fil. Eur., 270 (1867); Selaginella japonica Hort., Proc. Roy. Hort. Soc. 4: 136 (1864); Selaginella microstachya (Desv.) Hieron., Elmer's Leaflets Phil. Bot. 6: 1988. (1913); Selaginella microstachya Warb., Monsunia 1: 104, 116, no. 62 (1900); Selaginella pachystachys Koidz., Acta Phytotax. Geobot. 4: 226 (1935); Selaginella peltata Presl; Bot. Bem. 152 (1844); Selaginella pennula (Desv.) Spring, Bull. Acad. Brux. 10: 137 (1843); Selaginella pseudostauntoniana Pamp., Nuovo Giorn. Bot. Ital N. S., 18: 103 (1911); Selaginella warburgii Hieron., Elmer's Leaflets Phil. Bot. 6: 1988. (1913).

Perennial herb, robust, erect or ascending, without branches on the lower half, with a creeping shallow stoloniferous rhizome, fan-shaped frond, terrestrial, epiphythic or xerophytic, up to ca. $60 \mathrm{~cm}$ tall, $3-4 \mathrm{~cm}$ wide (including leaves), yellowish green, rolling up when dry. Stem hard, but easily broken, two types, i.e.: erect main stem and creeping subterranean rhizome. Main stems branched from half upward, dendritic, fan-shaped, pinnately branched, stramineous, unbranched main stem 20-60 cm long, 1-1.5 $\mathrm{mm}$ in diam. with several dormant buds or leaves; in lower part, terete, not sulcate, glabrous; leafy main stem including leaves $4-6 \mathrm{~mm}$ wide at middle, ultimate branches 2-3 $\mathrm{mm}$ wide including leaves. Rhizophores restricted to creeping rhizomes, at intervals. Leaves on the rhizome scale like, monomorphic, ovate, ciliate, sessile, apex acute, apprised or recurved, colorless to pale yellow or brown; Leaves of the half basal main stem monomorphic, ovate, clasping, nearly asymmetrical, appressed, 1-2 mm long, 1-1.8 mm wide, apex acute to attenuate, base truncate, auriculate or not, margin serrate to serrulate but lacerate with spinose at the auricule, arose and long ciliate towards apex. Leaves on the branches dimorphic, arranged in 4 lanes (2 lateral, 2 median), vein single, reaching the apex; lateral leaves lanceolate to ovate, contiguous or overlapping, slightly ascending, asymmetrical, 1-2.5 mm long, 0.3-1.5 mm wide, slightly carinate, ciliate near base, apex attenuate or acuminate, base cuneate or oblique with auriculate, vein single always curved and pointing to abaxial side, having 2 significant grooves beside the vein, adaxial blade raised and forming two-main-vein, margin denticulate or laciniate but spinose at the auricule; median leaves ovate on the main stem but elliptical or laceolate to ovate on the top branch, asymmetrical, 1.5-3 mm long, 1-2.5 mm wide, apex acute, base rounded to subcordate, twisting to form miniature auricle at the base, single vein, obscure, 1-2 longitudinal groove (s) at the adaxial surface beside the vein of median leaves on the top branch, having 2-3 grooves at the abaxial surface on the top branch, 2 beside the vein and 1, less significant or absent, inside the midrib, margin entire to serrate, laciniate at most basal part of margin, concentrated spinose at the miniature-auricle base, minutely ciliate, pointing upwards; axillary leaves ovate to cordate on first forked site but lanceolate to ovate at following forked site, nearly symmetrical, 1-2.5 mm long, 0.5-1.5 mm wide, apex acute or attenuate, base subcordate or cordate, exauriculate, margin serrate but laciniate at basal part, minutely ciliate or denticulate. Strobilus are solitary, terminal, tetragonal, compact, up to $2 \mathrm{~cm}$ long; sporophylls monomorphic.

Habitat and ecology: It was found on the rocky steep cliff, the steep wall of small-river bank, the edge of irigation canal near tobacco fields, the cliff of rainwater canal near tea gardens and vegetable fields; at altitude of 351-1493 m. asl.

Locality: Banjarnegara (Kalibening), Purbalingga (Mrebet), Purworejo (Bruno), Temanggung (Ngadirejo, Parakan), Wonosobo (Kretek)

Distribution: Bhutan, Cambodia, China (common), India, Indonesia, Japan (Honshu, Shikoku, Kyushu, Ryukyu Isl.), Korea, Laos, Myanmar, Nepal, Palau Isl., Sri Lanka, Philippines, Taiwan, Thailand, Vietnam. In Indonesia: Java, Lesser Sunda Isl. (Flores), Kalimantan, Sulawesi.

Specimens observed: ADS 97, ADS 38, ADS 415, ADS 517, ADS 1095, ADS 1096, ADS 1116, ADS 1139, ADS 1443, ? (BO!).

Note: In the study site, $S$. involvens is collected in lowland until highland areas of rocky sites. In Central Java, it is also found in the highland of Mt. Merapi (Setyawan et al. 2012) and Mt. Lawu (Setyawan et al. 2013); and the lower mountain of Ngelanggeran (300-500 m) (Setyawan 2012).

Selaginella opaca Warb., Monsunia 1: 108, 122, no. 112 (1900) (Figure 3.F)

Synonym: Selaginella cerebriformis Alderw., Bull. Jard. Bot. Buit. II, 7: 32-33 (1912); Selaginella mearnsii Hieron., Elmer's Leaflets Phil. Bot. 6: 2018, no. 16 (1913); Selaginella pervaga Hieron., Elmer's Leaflets Phil. Bot. 6: 2021, no. 17 (1913). 
Perennial fleshy herb, creeping, ascending, hanging, glabrous, up to ca. $2 \mathrm{~m}$ long, generally $0.5 \mathrm{~m}$. Stems creeping to ascending, usually fertile branches alternate on long fleshy main stem, 40-80 (-200) cm long, $3-8 \mathrm{~cm}$ wide (including leaves). Rhizophores at intervals stem, mostly near the base, originated from the dorsal side of stem, ca. 1-1.5 $\mathrm{mm}$ in diam. Leaves conspicuously dimorphic, but monomorphic on the main stem, oblong, asymmetrical, spaced farther apart than their width, midrib present. Leaves on the branches are dimorphic, arranged in 4 lanes (2 dorsal, 2 ventral), loosely arranged at long creeping stem but closely arranged at branches; lateral leaves ovate to oblong, asymmetrical, 2-5 mm long, 2-3 $\mathrm{mm}$ wide, apex acute, base rounded, margin serrulate to entire or minutely ciliate at the base, pointing outwards, imbricating at the ends of branches, vein single, obscure, not reaching the apex; median leaves ovate to oblong, asymmetrical, 1.5-3 mm long, 1-2 mm wide, apex caudate, base obliquely cordate or cordate, pointing upwards, imbricating at the ends of branches, margin serrulate or serrate, but entire at basal part, vein single not reaching the apex; axillary leaves ovate, entire, rounded or obtuse, symmetrical, 2.5-3.5 mm long, 1.5-2.5 mm wide, apex acute, base rounded, margin entire or serrulate at apical part. Strobilus solitary (rarely twin), terminal or lateral, tetragonal, up to more than $3.5 \mathrm{~cm}$ long; sporophylls monomorphic.

Locality: Banjarnegara (Batur, Wanayasa), Temanggung (Parakan), Pekalongan (Petungkriyono), Wonosobo (Garung, Kejajar, Kretek, Mojotengah).

Habitat and ecology: This species is typical of highland region. It is found on the banks of irrigation canals, water springs, creeks, roadside ditch, rainwater canals, river banks shaded by bamboo, waterfront of lake; in the fields of vegetables and medicinal plants, dry fields, and potato field; on the ground floor of pine forest, Albisia lophanta forest, and deforested areas; on the cliffs of roadsides village and main roads, vegetable fields and cemetery area; at altitude of993-2124m. asl.

Distribution: Indonesia, New Guinea, Philippines. In Indonesia: Java, Lombok, Moluccas (Ceram), Sumatra.

Specimens observed: ADS 76, ADS 84, ADS 86, ADS 88, ADS 89, ADS 171, ADS 181, ADS 183, ADS 189, ADS 193, ADS 199, ADS 208, ADS 216, ADS 224, ADS 238, ADS 246, ADS 253, ADS 283, ADS 305, ADS 308, ADS 339, ADS 340, ADS 347, ADS 355, ADS 357, ADS 362, ADS 373, ADS 374, ADS 399, ADS 402, ADS 411, ADS 434, ADS 437, ADS 442, ADS 444, ADS 520, ADS 538, ADS 542, ADS 543, ADS 550, ADS 1089, ADS 1091, ADS 1093, ADS 1094, ADS 1097, ADS 1099, ADS 1102, ADS 1119, ADS 1141, ADS 1408, ADS 1412, ADS 1415, ADS 1418, ADS 1422, ADS 1431, ADS 1433, ADS 1436, ADS 1438, ADS 1440, ADS 1445, ADS 1466, JA Lorzing 416 (BO!), R Brinkman 165 (BO!)

Note: This species is mostly cohabit withS. remotifolia; a habitat that covered by $S$. opaca is almost certainly covered by $S$. remotifolia, and vice versa. Both of these species can grow creeper, but $S$. opaca tend to ascending. $S$. opaca has a larger stem and juicy, while $S$. remotifolia has a small wiry stem with unlimited growth (when the main stem dies, the branches developing into a new individual). In the rainy season, the presence of $S$. remotifolia is more abundant due to its ability to grow in agricultural lands, while $S$. opaca is likely removed by the farmer. On the cliff of vegetable fields, a clump of this species can grow up to $2 \mathrm{~m}$ long and $1 \mathrm{~m}$ wide; they grow hanging on the cliffs of fields. In the Dieng plateau, it was also subjected to frost that caused the tops of branches bleach and die, but it will grow back new branches.

Selaginella ornata (Hook \& Grev.) Spring, Bull. Acad. Brux. 10: 232 (1843) (Figure 3.G-H, Figure 4)

Basionym: Lycopodium ornatum Hook. \& Grev., Enum. Fil. Hook. Bot. Misc. 3: 108 (1883).

Synonym: Lycopodium atro-viride Wall. ex Blume, Enum. Pl. Jav. 2: 269 (1830) (deser.) [non Wall. ex Hook. 1831]; Selaginella balica Alderw., Bull. Jard. Bot. Buit. III, 2: 181 (1920); Selaginella blumei Spring, Bull. Acad. Brux. 10. 143 (1843); Selaginella brachystachya var. ornata (Hook. \& Grev.) Baker, Jour. Bot. 23: 180 (1885); Selaginella duriuscula Alston ex Knox; Trans. Bot. Soc. Edinb. 35: 269 (1950); Selaginella fimbriata Spring, Monogr. Lyc. II: 258, no. 198 (1850); Selaginella fimbriata var. grandifolia Alderw., Bull. Jard. Bot. Buit. III, 2: 183 (1920); Selaginella fimbriata var. polyura Warb., Monsunia 1: 110, 127 (1900); Selaginella geniculata var. tomentosa (Spring) Baker, Jour. Bot. 23: 121 (1885); Selaginella javanica Klotzsch ex Zoll., Syst. Verz. 1: 50 (1854); Selaginella javanica var. gracilis A. Br., ex Alderw., Bull. Jard. Bot. Buit. II, 7: 34 (1912); Selaginella petrophila Alderw., Bull. Jard. Bot. Buit. III, 5: 234 (1922); Selaginella polita Ridl., Jour. Fed. Mal. States Mus. 6: 202, no. 348 (1915); Selaginella rabenavii Hieron., Engl. \& Prantl, Nat. Pfl. 1 (4): 694, no. 244. [1901] 1902; Selaginella sclerophila Alderw., Bull. Jard. Bot. Buit. III, 2: 182 (1920); Selaginella subfimbriata Alderw., Bull. Jard. Bot. Buit. II, 1: 26 (1911); Selaginella subfimbriata var. koordersii Alderw., Bull. Jard. Bot. Buit. III, 1: 26 (1911); Selaginella subfimbriata var. polyura (Warb.) Alderw., Bull. Jard. Bot. Buit II, 7: 34 (1912); Selaginella subfimbricata var. backeri Alderw., Bull. Jard. Bot. Buit. III. 1: 26 (1911); Selaginella tomentosa Spring, Monogr. Lyc. II: 231, no. 168 (1850); Selaginella varians Alderw., Bull. Jard. Bot. Buit. III, 5: 237 (1922); Selaginella varians f. sciaphila Alderw., Bull. Jard. Bot. Buit. III, 14: 237 (1922); Selaginella xerophila Alderw., Bull. Jard. Bot. Buit. III, 2: 182 (1920).

Perennial herb, creeping to ascending or sub erect, brittle, glabrous, $20-40 \mathrm{~cm}$ long, green to brownish (reddish) green. Stems oval, flattened, or sub quadrangular, sulcate or not, glabrous, branched from near base upward, green, stramineous or brownish, fragile, very easily broken,0.7-1.5 $\mathrm{mm}$ in diam. $1-3 \mathrm{~cm}$ wide (including leaves). Rhizophores at intervals throughout length of creeping stem and branches, rarely on upper part, originated from ventral side in axils of branches, ca. 0.5-1 $\mathrm{mm}$ in diam, brownish or green. Leaves dimorphic, arranged in 4 lanes ( 2 dorsal, 2 ventral), densely arranged throughout the stem and imbricating at top of branches; lateral leaves oblong to oblong-falcate, denticulate to 


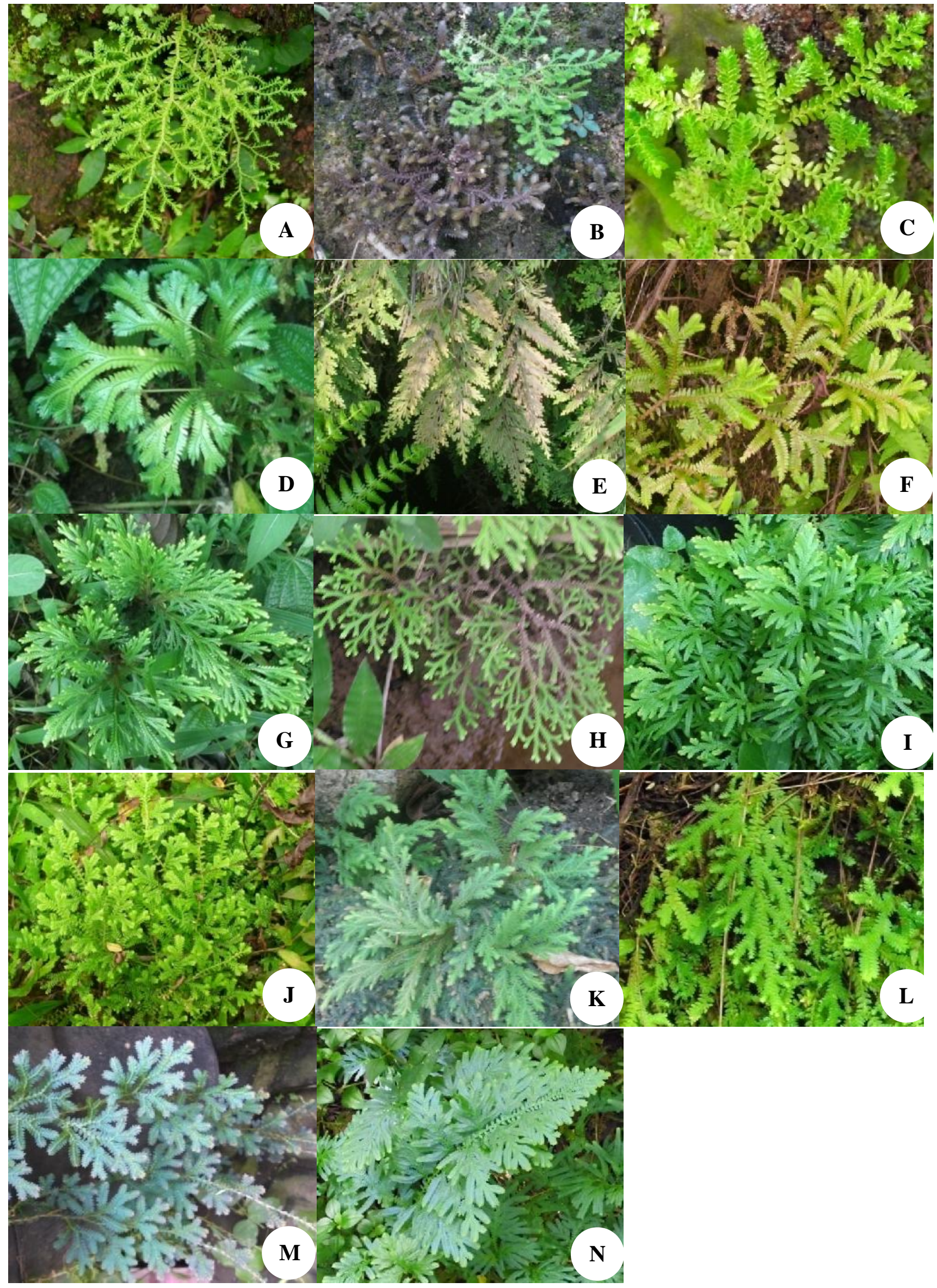


Figure 3. Species diversity of selaginellas in Southern Java and the surrounding; A. S. aristata, B. S. ciliaris (brown and green colors), C. S. ciliaris, D. S. intermedia, E. S. involvens, F. S. opaca, G. S. ornata, H. A variant of S. ornata, I. S. plana, J. S. remotifolia, K. S. repanda, L. S. singalanensis, M. S. uncinata, and N. S. willdenowii

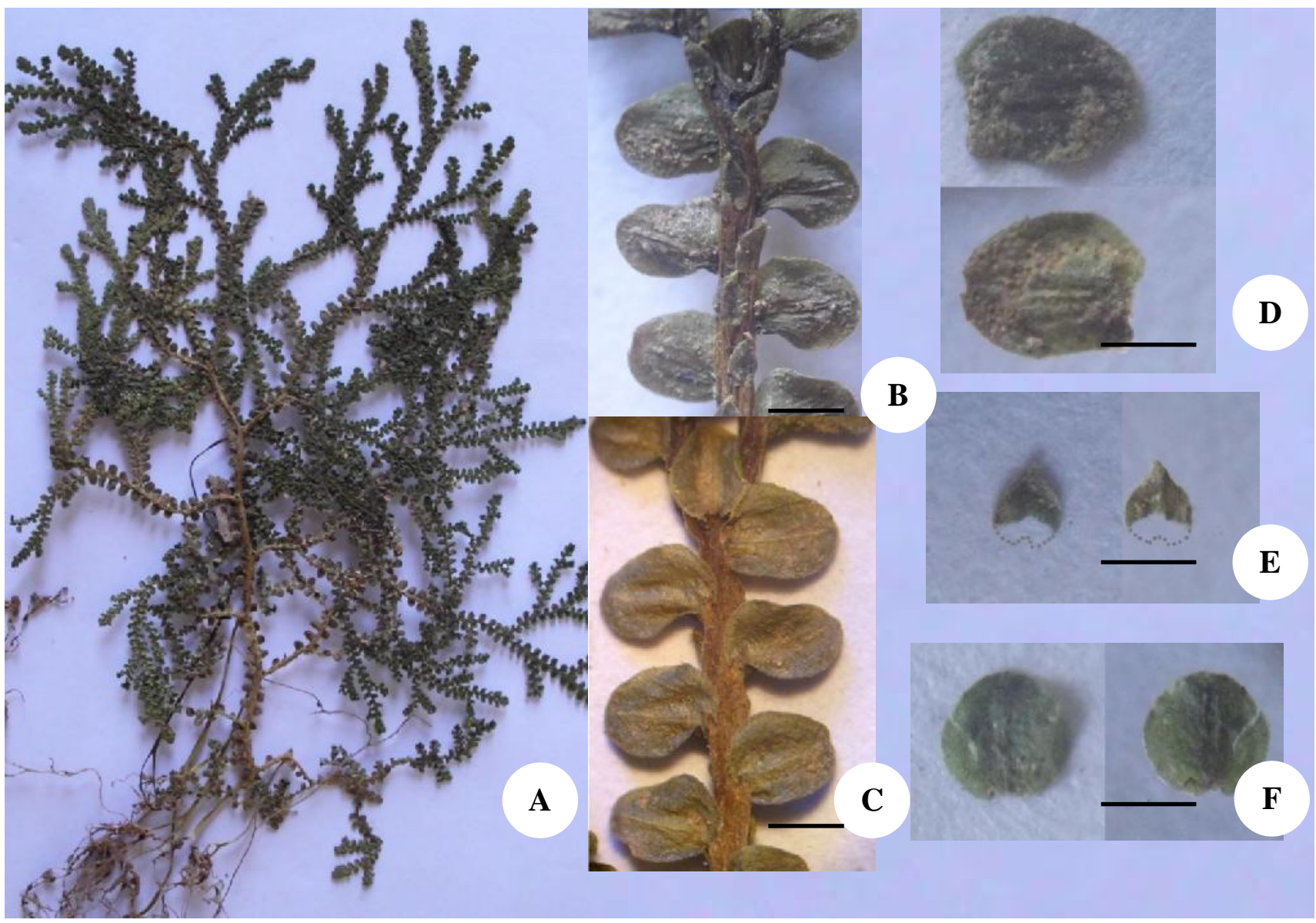

Figure 4. A morphological variant of S. ornata; A. Habit. B. Adaxial stem, with lateral and median leaves. C. Abaxial stem, with lateral and axillary leaves. D. Lateral leaves. E. Median leaves. F. Axillary leaves. Note: Strobilus has not developed yet, but some leaves on the top branches clumped. Bar $=1 \mathrm{~mm}$

dentate, exauriculate, asymmetrical, on main stem larger than on branches, distant or contiguous, spreading, 2-3 mm long, 1-2 mm wide, apex obtuse, acuminate or acute, and prickly tip, base rounded to truncate, basiscopic base decurrent, margin entire; acroscopic base rounded, overlapping stem and branches, margin denticulate in basal half, vein single not reaching the apex; median leaves contiguous or imbricate, ovate, denticulate to dentate, carinate, with arista often more than half the lamina length, asymmetrical, 1.5-3.5 mm long, 0.5-1.5 mm wide, apex acute or aristate, prickly tip, base obtuse or rounded, attenuate, vein single not reaching the apex, margin minutely denticulate to entire; axillary leaves ovate, lanceolate or subcordate, exauriculate, imbricating, asymmetrical, $1-1.5 \mathrm{~mm}$ long, 0.5-1 mm wide, apex acute, base rounded, margin entire. Strobilus solitary or twins, terminal, compact, dorsiventrally complanate, bisymmetrical, upper-plane sporophylls longer than lower- plane sporophylls, up to more than $1 \mathrm{~cm}$ long; sporophylls strongly dimorphic, spores pale yellow to reddish brown.

Locality: Banjarnegara (Batur, Banjarmangu, Kalibening, Madukara, Pagentan, Sigaluh), Magelang (Kajoran), Pekalongan (Paninggaran), Purworejo (Kaligesing), Wonosobo (Garung, Kalikajar, Kaliwiro, Kepil, Kertek, Mojotengah, Sapuran, Selomerto, Watumalang, Wonosobo-city).

Habitat and ecology: It was found around the water springs and irrigation canals, river bank, small road ditch, footpath, vegetable and dry fields; in the pine forest, bamboo, albizia, guava and cloves fields; on the steep cliffs of vegetable fields, pine and agathis forest, rice fields, roadside of villages and main roads; creeks, small rivers and irrigation canals; on the cemetery hill; at altitude of 222-1457 m. asl.

Distribution: Cambodia, India, Indonesia, Malaysia (Peninsular), Philippines, Thailand, Vietnam. In Indonesia: 
Java, Kalimantan, Lesser Sunda Isl. (Bali, Flores, Lombok), Sumatra.

Specimens observed: ADS 76, ADS 77, ADS 84, ADS 86, ADS 88, ADS 89, ADS 164, ADS 174, ADS 176, ADS 187, ADS 191, ADS 196, ADS 205, ADS 213, ADS 221, ADS 235, ADS 243, ADS 249, ADS 279, ADS 300, ADS 303, ADS 334, ADS 335, ADS 342, ADS 350, ADS 352, ADS 357, ADS 368, ADS 369, ADS 394, ADS 397, ADS 406, ADS 429, ADS 432, ADS 437, ADS 439, ADS 514, ADS 531, ADS 535, ADS 536, ADS 542, ADS 1062, ADS 1064, ADS 1066, ADS 1067, ADS 1070, ADS 1072, ADS 1117, ADS 1120, CA Backer 21865 (BO!), Koorders 27142 (BO!).

Note: This species has a morphological appearance that is quite diverse and is generally characterized by a trunk which is very brittle. General appearance of $S$. ornata is brownish, but there are also variants of the green without brown nuances. In the study, $S$. ornata has a fairly wide distribution, ranging from the hilly area around the Wadaslintang reservoir in southern part of Wonosobo up to Dieng Mountains.

In this study, We have discovered a variant of Selaginella which the leaves (trophophylls) are round, either the lateral, median, or axillary leaves (apex long acuminate in this ones); it also occurs in the leaves at the tip of branches which expected to be developed into strobilus (sporophylls) (Figure 3.H and 4). The specimen is the only one, several intensive field surveys at the discovery site (Kaliwiro of Wonosobo District) and around was not able to find the same specimen, thus that the specimen was thought to be a variant of the species present at that location. The most similar species is $S$. ornata, although it was also found $S$. aristata, $S$. ciliaris, and $S$. plana.

Selaginella plana (Desv. ex Poir.) Hieron., Nat.

Pflanzenfam. 1 (4): 703 (1901) (Figure 3.I)

Basionym: Lycopodium planum Desv. ex Poir., Lamarck, Encycl. Suppl. 3: 554, no. 98. [1813] 1814;

Synonym: Lycopodium canaliculatum var. pallidius Hook. \& Arn., Bot. Beechey's Voy., 255 (1836)-1840; Lycopodium durvillaei Bory in Duprey, Voy. Bot. 1: (247, no. 12), t. 25 (1829) (excl. descr.); Lycopodium nemorum Desv., Ann. Soc. L. Paris, 6: 186, no. 83 (1827); Lycopodium pellucidum Desv. in Poir., Lamarck, Encycl. Suppl. 3: 552, no. 88. [1813] 1814; Selaginella bellula T. Moore, Gard. Chron. 11: 173, f. 25 (1879) [non Ces. 1876]; Selaginella caudata var. durvillaei (Bory) Spring, Monogr. Lyc. II: 141 (1850); Selaginella caudata var. guichenotii Spring, Monogr. Lyc. II: 140 (1850); Selaginella durvillaei (Bory) A. Br. ex Kuhn, Verh. K. K. Zool. Bot. Ges. Wine 19: 585, no. 131 (1869); Selaginella guichenotii (Spring) Hieron., Engl. \& Prantl, Nat. Pfl. 1 (4): 701, no. 346. [1901] 1902; Selaginella herpocaulos var. acuminata Alderw., Bull. Jard. Bot. Buit. III, 2: 185 (1920); Selaginella inaequalifolia var. perelegans (Moore) Baker, Jour. Bot. 23: 4 (1885); Selaginella perelegans T. Moore, Gard. Chron. 11: 533 (1879).

Common name: Asian spike moss
Perennial herb, stout, sub erect to ascending with stoloniferous rhizome, without branches on the lower part, ascending from a subterranean trailing base, glabrous, fanshaped, green. Stems suberect to ascending, branches on the upper half, up to $80-100 \mathrm{~cm}$ long, $3-10 \mathrm{~cm}$ wide (including leaves);subterranean stems (rhizome) shallowly radiating. Rhizophores present sometimes at the branching stem, originated from the dorsal side of stem at the branch site, ca. $1-1.5 \mathrm{~mm}$ in diam. Leaves on the lower part and main stem are monomorphic, well spaced, appressed, 1.5-3 mm long, 1-2 mm wide, upper part slightly spreading, ovate, asymmetrical, apex acuminate or acute, but rounded tip, margin translucent, entire. Leaves on the branches are dimorphic, arranged in 4 lanes (2 dorsal, 2 ventral), loosely arranged at lower stem but closely arranged at branches; lateral leaves are oblong to ovate, asymmetrical, 2-4.5 mm long, 2-3 mm wide, apex acuminate-acute to rounded, but rounded tip, sessile, vein single, obscure, not reaching the apex, base truncate and rounded, upper base with a spur-like lobe which overlaps the stem, margin transparent, entire; median leaves are ovate to oblong, asymmetrical, 1.5-3 mm long, 1-2 mm wide, apex acuminate-acute to obtuse-rounded, but rounded tip, sessile, vein single, obscure not reaching the apex, base truncate and rounded, margin transparent, entire; axillary leaves are ovate or obovate-oblong, asymmetrical, 2.5-3.5 $\mathrm{mm}$ long, $1.5-2.5 \mathrm{~mm}$ wide, apex acute to slightly acuminate, minutely ciliate, base rounded, margin entire. Strobilus are solitary, terminal, tetragonal, up to more than $3 \mathrm{~cm}$ long; sporophylls monomorphic.

Locality: Banjarnegara (Banjarmangu, Banjarnegaracity, Bawang, Kalibening, Madukara, Pagedongan, Pagentan, Sigaluh, Wanadadi), Kebumen (Buayan, Gombong, Karanggayam, Karangsambung, Kebumen-city, Prembun, Sempor), Kulonprogo (Girimulyo), Magelang (Kajoran), Pekalongan (Paninggaran), Purbalingga (Bobotsari, Karangmoncol, Rembang), Purworejo (Kaligesing), Temanggung (Bejen, Candiroto, Jumo, Kandangan), Wonosobo (Kaliwiro, Kepil, Kertek, Sapuran, Selomerto, Wadaslintang, Wonosobo-city)

Habitat and ecology: It was found around the water spring, riverside creeks and small rivers, small and large irrigation canals, fish pond, road ditch; roadside village and main road, home garden; dry fields and paddy fields; in the coffee plantations, salak, coconut, albizia, bamboo; in the pine, agathis and mixed forests; cliffs of the rivers bridges, water springs, creeks and small rivers, irrigation canals; rock cliffs and moist crevices of limestone, cliffs of the roadside villages and main street, cliff in the home garden, salak, acacia, albizia, bamboo, teak plantations, pine, agathis, mixed forests, dry fields vegetable and rice field; among the rocks and buildings around the tomb; on agricultural land, is considered a weed species that tend to be cleaned; at altitudes of $19-1020 \mathrm{~m}$. asl.

Distribution: Indonesia, Malaysia (Peninsular). In Indonesia: Java, Lesser Sunda Isl. (Bali, Flores, Sumbawa, Solor, Timor), Sulawesi, Sumatra, Moluccas (Ambon, Banda, Buru, Ceram, Kei Isl., Ternate). Introduced to Asia: India, Philippines,Taiwan. Introduced to America: Barbados, Brazil, British Guyana, Colombia, Costa Rica, 
Dominica, Ecuador, Honduras, Jamaica, Martinique, Panama, Puerto Rico, USA (Florida), St. Kitts, St. Thomas, Trinidad. Introduced to Africa: Tanzania.

Specimens observed: ADS 74, ADS 75, ADS 79, ADS 80, ADS 81, ADS 82, ADS 98, ADS 99, ADS 100, ADS 169, ADS 170, ADS 172, ADS 173, ADS 174, ADS 175, ADS 177, ADS 178, ADS 179, ADS 184, ADS 185, ADS 209, ADS 210, ADS 214, ADS 217, ADS 225, ADS 226 , ADS 237, ADS 241, ADS 242, ADS 245, ADS 251, ADS 252, ADS 312, ADS 313, ADS 315, ADS 318, ADS 321, ADS 325, ADS 326, ADS 334, ADS 341, ADS 348, ADS 361, ADS 365, ADS 368, ADS 371, ADS 377, ADS 397, ADS 400, ADS 403, ADS 406, ADS 408, ADS 523, ADS 535, ADS 539, ADS 736, ADS 737, ADS 739, ADS 741, ADS 1054, ADS 1056, ADS 1058, ADS 1060, ADS 1062, ADS 1064, ADS 1066, ADS 1068, ADS 1069, ADS 1071, ADS 1072, ADS 1078, ADS 1079, ADS 1082, ADS 1086 , ADS 1088, ADS 1090, ADS 1092, ADS 1101, ADS 1103 , ADS 1107, ADS 1118, ADS 1130, ADS 1132, ADS 1133, ADS 1135, ADS 1136, ADS 1137, ADS 1140, ADS 1424, ADS 1427, ADS 1429, ADS 1430, ADS 1432, ADS 1434, ADS 1439, ADS 1441, ADS 1444, ADS 1446, ADS 1447, ADS 1448, ADS 1451, ADS 1454, ADS 1455, ADS 1457, ADS 1458, ADS 1459, ADS 1461, ADS 1462, ADS 1464, ADS 1465, ADS 1467, Banjoemas 2117 (BO!), Banjoemas 2480 (BO!), R. Brinkman 28 (BO!), ? 790 (BO!).

Notes: It is originally low-lying selaginellas species that most widely spreading, and $1020 \mathrm{~m}$ is the highest point reached in the study area. To date, the highest point that can be reached in Java is $1150 \mathrm{~m}$. asl. in Mt. Lawu (Setyawan et al. 2013) and $1200 \mathrm{~m}$. asl. in Mt. Merapi (Setyawan et al. 2012).

Selaginella remotifolia Spring, Miq. Pl. Jungh. 3: 276, no. 5 (1854) (Figure 3.J)

Basionym: Lycopodioides remotifolia (Spring) H.S. Kung, Fl. Sichuanica 6: 65-67 (1988)

Synonym: Selaginella involucrata Warb., Monsunia 1: 102, 113, no. 28 (1900); Selaginella japonica Miq., Ann. Mus. Bot. Ludg. Bat. 3: 185 (1867); Selaginella kelungensis Hayata; Icon. P1. Formosan 7: 97-98 (1918); Selaginella kraussiana auct. non (Kunze) A. Braun: French. \& Sav., Enum. Pl. Jap. 2 (1): 200 (1877); Selaginella remotifolia Spring var. japonica (Miq.) Koidz., Acta Phytotax. Geobot. 4: 228 (1935); Selaginella utchinensis Koidz., Acta Phytotax. Geobot. 4: 229 (1935)

Perennial herb, wiry, creeping, glabrous, branched from near base upward, several fertile branches alternate on long main stem, not obviously articulate, up to $100 \mathrm{~cm}$ long, green to stramineous stem. Stems creeping, oval or terete, sulcate, stramineous, $0.5-1.5 \mathrm{~cm}$ wide (including leaves). Rhizophores at the branching stem, throughout length of creeping stem and branches, originated from the dorsal side in axil of stem branches, ca. $0.5 \mathrm{~mm}$ in diam. Leaves on the main stem monomorphic, decussate, lanceolate, acuminate, asymmetrical, spaced farther apart than their width, midrib present. Leaves on the branches dimorphic, arranged in 4 lanes ( 2 dorsal, 2 ventral), loosely arranged at the long creeping main stem but closely arranged at branches, those on main stems slightly larger than those on branches; lateral leaves contiguous, lanceolate to ovate, distant or approximate, spreading, asymmetrical, 1.5-3 mm long, 1-2 $\mathrm{mm}$ wide, apex acute to acuminate, vein single, obscure not reaching the apex, base rounded, margin serrulate, entire or minutely ciliate, denticulate, pointing outwards, fertile branches erect; median leaves lanceolate to ovate, asymmetrical, 1.5-3 mm long, 0.5-1 mm wide, not carinate, apex attenuate acuminate, or caudate, base obliquely cordate or cuneate, uni-auriculate, approximate or imbricate at ends of branches, margin serrulate or serrate, but sub entire or minutely denticulate at abaxial medium and basal part, vein single; axillary leaves ovate, broadly ovate, entire, rounded or obtuse, symmetrical, 1.5-2.5 mm long, 1-1.5 mm wide, apex acute, base exauriculate, margin entire, slightly denticulate or loosely serrulate at apical part. Strobilus solitary, terminal or lateral, compact, tetragonal, up to more than $0.5-2 \mathrm{~cm}$ long; sporophylls monomorphic.

Locality: Banjarnegara (Batur, Pagentan, Pejawaran, Wanayasa), Pekalongan (Petungkriyono), Temanggung (Parakan), Wonosobo (Garung, Kejajar, Kretek, Mojotengah, Watumalang, Wonosobo-city)

Habitat and ecology: It was found on the banks and cliffs of creeks, small rivers, irrigation canals, ditch of the vegetable and tobacco fields and mixed forest, waterfront of lake, steep cliff of waterfall; on the intensive agricultural field shaded by paranet; the steep cliffs of dry field, on the edge of field and among vegetable plants, near the tea garden; in the agroforesty of albizia; Paraserianthes lophantha forest and deforested forests (reforestation); roadside and cliffs of the dirt road, villages road, and main roads, cliff near residential area; at altitudes of $1150-2070$ m. asl.

Distribution: China (common), Indonesia, Japan (Hokkaido, Honshu, Kyushu, Shikoku, Ryukyu Isl.), Korea, Malaysia, Myanmar, New Guinea, Philippines, Taiwan. In Indonesia: Java, Sumatra.

Specimens observed: ADS 21, ADS 23, ADS 40, ADS 42, ADS 83, ADS 85, ADS 87, ADS 90, ADS 93, ADS 94, ADS 95, ADS 96, ADS 180, ADS 192, ADS 198, ADS 256, ADS 257, ADS 259, ADS 260, ADS 261, ADS 263, ADS 264, ADS 282, ADS 300, ADS 304, ADS 352, ADS 356, ADS 412, ADS 416, ADS 436, ADS 439, ADS 443, ADS 518, ADS 544, ADS 547, ADS 553, ADS 555, ADS 556, ADS 558, ADS 559, ADS 562, ADS 565, ADS 566, ADS 1122, ADS 1124, ADS 1127, ADS 1125, ADS 1128, ADS 1057, ADS 1420, ADS 1409, ADS 1413, ADS 1416, Ruttner 276 (BO!), W Meijer 2755 (BO!).

Note: This species has the same habitat as $S$. opaca, but it is more easily found in farm fields, due to the small size and creeping stems that make it difficult to be eradicated and become weeds in the uplands; even it can compete with the ground grass.

Selaginella repanda (Desv. ex Poir.) Spring, Gaud. Voy. Bonite Bot. 1: 329 (1846) (Figure 3.K)

Basionym: Lycopodium repandum Desv. ex Poir.; Lamarck, Encycl. Suppl. 3: 558. [1813] 1814;

Synonym: Lycopodioides barbata (Spring ein. Baker) Kuntze, Rev. Gen. Pl. 1: 826 (1891); Lycopodioides compta (Hand.-Mazz.) H.S.Kung, Fl. Sichuanica 6: 76 
(1988); Lycopodium barbatum Kaulf., Enum. Fil. 18 (1824); Lycopodium plumosum Dill., Hist. Musc., t. 66, f. 10 (1714); Lycopodium radicatum Hook. \& Grev. Bot. Misc. 2: 397 (1831); Lycopodium semicordatum Wall. ex Hook. \& Grev., Enum. Fil., Hook. Bot. Misc. 2: 396, no. 158 (1831); Lycopodium tetragonostachyum var. major Hook. \& Grev., Enum. Fil., Hook. Bot. Misc. 2: 389 (1831); Lycopodium tetragonostachyum Wall., Cat. no. 124 (1829), ex Hook. \& Grev., Enum. Fil., Hook. Bot. Misc. 2: 389, no. 129. (1831); Sclaginella plumosa var. hamiltoni Baker; Jour. Bot. 21: 145 (1883); Selaginella barbata (Kaulf.) Spring, Bull. Acad. Brux. 10: 226, no 101 (1843); Selaginella ceratocaulos Alderw., Bull. Jard. Bot. Buit. II, 16: 41 (1914); Selaginella ceratocaulos f. madurensis Alderw., Bull. Jard. Bot. Buit. II, 13: 434 (1914); Selaginella ceratocaulos f. typica Alderw., Mal. Fern Allies 239 (1915); Selaginella ceratocaulos var. kangeanensis Alderw., Bull. Jard. Bot. Buit. III, 5: 232 (1922); Selaginella compta Hand.-Mazz., Sumb. Sin. 6: 9 (1929); Selaginella ganguliana Dixit, Bull. Bot. Surv. India 26: A-I (1984) [fide Fraser-Jenkins (1997)]; Selaginella henryi Koidz., Fl. Symb. Orient. Asiat. 85 (1930); Selaginella implexa Scott, Jour. Agr. Hort. Soc. India N. S. 1 (2): 262 (1868); Selaginella implexa Scott, List Higher Crypt., 64 (1868); Selaginella mongholica auct. non Ruhr.: A. Henry, trans. Asiat, Soc, Japan 24 (Suppl.): 117 (1896); Selaginella plumosa f. tetragonostachya (Wall.) Haines, Bot. Brit. \& For (Bot. Bih. Or.), p. 1224 (1924); Selaginella plumosa var. radicata (Hook. \& Grev.) Warb., Monsunia I: 102 (1900); Selaginella pyrrhopus Spring, Monogr. Lyc. II: 114-115 (1850); Selaginella radicata (Hook. \& Grev.) Spring, Mem. Acad. R. Sci. Belgique 24 (2): 114 (1850); Selaginella subcaulencens auct. non Hayata: Tak. Ito, Icon. Taiwan Pl. Suppl. Vol. 4 (1928); Selaginella suberecta Baker, Jour. Bot. 22: 245, no. 146 (1884); Selaginella tetragonostachya (Wall.) Spring, Bull. Acad. Brux. 10: 234, no. 163 (1843); Selaginella vaginata Spring, Mem. Acad. R. Sci. Belgique 24: 87 (1850).

Perennial (or annual) herb, terrestrial or epiphytic, ascending from decumbent base, ciliate, brownish (reddish) green leaves with reddish green to brown stem, scabrous; multiple branched at creeping stem, forming ascending dendritic, fan-shaped stem. Stems stramineous, oval or terete, 2-forked, consists of two types, i.e. creeping on the ground (vegetative), multiple branched, forming diffuse mats, up to ca. $45 \mathrm{~cm}$ long, 3-4 $\mathrm{mm}$ wide (including leaves); or decumbent to ascending (generative), dendritic, fan-shaped, especially at the mature ones, ca. 10-30 cm long, 4-5 mm wide (including leaves). Rhizophores present at creeping stem (rhizome), and sometimes also on base of ascending ones, originated from ventral side in axils of branching stem, ca. $1 \mathrm{~mm}$ in diam. Leaves dimorphic, those on main stem larger than those on branches, arranged in 4 lanes (2 lateral, 2 median), sparsely arranged at main stem but imbricate at the branches, vein single; lateral leaves asymmetrical, approximate, spreading, oblong-falcate, 2-3 $\mathrm{mm}$ long, 1-1.5 mm wide, apex acute, base rounded, margin ciliolate, minutely denticulate to apex, single vein; median leaves smaller than the lateral ones, asymmetrical, approximate, obliquely ovate at branches, 1-2 mm long,
0.5-1 mm wide, not carinate or slightly carinate, apex long acuminate to shortly aristate, base obliquely subcordate, not peltate, margin long ciliate (denticulate to apex), reflexed, parallel to axis; axillary leaves ovate or ovatelanceolate, less or more symmetrical 2-3 mm long, 1-1.5 $\mathrm{mm}$ wide, apex acute or acuminate, base rounded-obtuse, exauriculate, margin ciliolate, single vein. Strobilus solitary, terminal, compact, sub tetragonal or subcomplanate, 2-6 mm long; sporophylls submonomorphic or sometimes dorsal sporophylls longer, spores orange-brown to bright yellow.

Locality: Kebumen (Buayan, Karanggayam, Prembun, Sempor), Purbalingga (Bobotsari)

Habitat and ecology: It is rare and usually found on the humid rocky cliffs, such as small river bank, moist crevices of limestone, sandstone cliffs of main road, roadsides and riverbanks shaded by bamboo, near ditch in the albizia fields; on the walls of cemetery and gravestones; at altitudes of 19-214 m. asl.

Distribution: Bhutan, Cambodia, China (common), India (Uttar Pradesh, Madhya Pradesh, Andhra Pradesh, Karnataka, Sikkim, Tamil Nadu), Indonesia, Laos, Malaysia (Perlis), Myanmar, Nepal, Philippines, Taiwan, Thailand, Vietnam. In Indonesia: Java, Lesser Sunda Isl. (Sumbawa, Timor), Sumatra.

Specimens observed: ADS 322, ADS 323, ADS 1053, ADS 1067, ADS 1080, ADS 1081, ADS 1138, ADS 1449, ADS 1450, and ADS 1453.

Note: In Java, It is thought to be typical species of rocky lowland to moderate hills, and dry climates (xerophytic).Spores are usually bright yellow, which distinguishes it from other selaginellas.

\section{Selaginella singalanensis Hieron., Hedwigia 50: 18, no. 12} (1910) (Figure 3.L)

Synonym: Selaginella flabelliformis Alderw., Bull. Jard. Bot. Buit. III, 2: 181 (1920); Selaginella junghuhniana Spring, Miq. Pl. Jungh. III: 276, no. 7 (1854); Selaginella modica Alderw., Bull. Jard. Bot. Buit. Ill, 5: 236 (1922)

Perennial herb, creeping, tender, in humid environments, growing all year round but mostly rainy season, yellowish green in general appearance. Stems creeping, irregular lateral branches, rooting at intervals, attached to the ground, very soft and very thin, $20-25 \mathrm{~cm}$ long, $1-3 \mathrm{~cm}$ wide (including leaves).Rhizophores at branching stem, originated from the dorsal side of stem at the branch site, ca. $0.5 \mathrm{~mm}$ in diam. Leaves are dimorphic, very soft, arranged in 4 lanes ( 2 dorsal, 2 ventral), densely arranged at thorough stem and imbricating at top of branches; lateral leaves are oblong, imbricating, asymmetrical, 1.5-2.5 mm long, 0.5-1.5 mm wide, apex acute, vein single not reaching the apex, base rounded, margin entire; median leaves oblong-ovate, dentate, exauriculate, imbricating, asymmetrical, 0.5-1.5 mm long, $0.5 \mathrm{~mm}$ wide, apex acute, base rounded, margin entire, vein single not reaching the apex; axillary leaves ovate, exauriculate, imbricating, asymmetrical, 0.5-1.5mm long, $0.5 \mathrm{~mm}$ wide, apex acute, base rounded, margin entire. Strobilus solitary, terminal, loosely, bisymmetrical, upper- 
plane, up to more than $1 \mathrm{~cm}$ long; upper-plane sporophylls longer than lower-plane.

Locality: Temanggung (Parakan)

Habitat and ecology: Locally not rare, also present somewhere else in the mountains. Prostate and at regular intervals rooting. The bottom lager is usually pressed to the ground. Usually often and regularly branching, 10- $30 \mathrm{~cm}$ long, often green, yellow or red brown, probably due to the drought. Very different [unreadable] spore careering parts which are not very visible; at altitude of $1800 \mathrm{~m}$. asl. (from the herbarium label of JA Lorzing 490, 18 June 1912 (BO!).

Distribution: Indonesia (Java, Sumatra)

Specimens observed: ADS 467, JA Lorzing 490 (BO!).

Selaginella uncinata (Desv. ex Poir.) Spring, Bull. Acad. Roy. Sci. Brux. 10: 141. 1843. (Figure 3.M)

Basionym: Lycopodium uncinatum Desv. ex Poir., Lamarck, Encycl. Suppl. 3: 558 (1814).

Synonym: Lycopodioides uncinata (Desv.) Kuntze, Rev. Gen. Pl. 1: 825 (1891); Lycopodium aristatum Roxb., Hort. Beng., 75 (1824) [nomen, non Hook. \& Bak]; Lycopodium caesium Hort. ex Anon., Ann. Hort. Soc (1847): 361, descr; Lycopodium dilatatum Hook. \& Grev., Enum. Fil., Hook. Bot. Misc. 2: 394, no 149 (1831); Selaginella aristata (Roxb.) Scott; List Higher Crypt., 64 (1868); Selaginella caesia Hort. ex A. Br., Ind. Sem. Hort. Berol. 23 (1860); Selaginella caesia Kunze, Linnaea 20: 2 (1847) [nom. nud.], Selaginella caesia var. violacea Hort. ex A. Br., Ind. Sem. Hort. Berol. 23 (1860); Selaginella eurystachya Warb., Monsunia 1: 105, 119, no. 79 (1900).

Common name: blue spike moss

Perennial herb, wiry, pliant, creeping, fan-shaped branching, up to ca. $75 \mathrm{~cm}$ long, forming diffuse mats. Stems are long-creeping, branched, 3-forked branching, flat or hanging, not articulate, glabrous with a wiry, pliant main stem, 1-1.5 mm diam., 4-5 cm wide (including leaves). Rhizophores are axillary at intervals, mostly at main stem base near to the ground, 0.3-0.5 $\mathrm{mm}$ diam. Leaves are dimorphic, delicate, papery, iridescent, green to metallic blue-green, single vein, arranged in 4 lanes (2 lateral, 2 median); lateral leaves are distant, ovate to oblong, asymmetrical, 2-3mm long, 1.5-2 $\mathrm{mm}$ wide, basiscopic base with small auricle, acroscopic base overlapping stem; base rounded or cordate, apex acute to obtuse, margins conspicuously transparent, entire, single vein reaching the apex, keeled; median leaves are ovate to lanceolate, asymmetrical, 2.5-4 $\mathrm{mm}$ long, 1.5-2 $\mathrm{mm}$ wide, base auriculate, apex acute to acuminate, margins transparent, entire, single vein; axillary leaves are present at branch forks, inserted at the ventral side of the stem, broadly ovate, more equally sided,3-4 mm long, 2-3 mm wide, single vein, base rounded to subcordate, apex acute to acuminate, margin transparent, entire, keeled. Strobilus is solitary, terminal, tetragonal, up to ca. $0.5-2 \mathrm{~cm}$ long and distinctly 4-keeled.

Habitat and ecology: It was only found once on the steep cliffs near the small river banks, covered/under the bushes; at altitude of $708 \mathrm{~m}$. asl. In the experimental garden, it can grow well, forming a thick mass in the floor and walls, hanging on hanging pots, fill the empty floor between the potted plants, very suitable as land cover plant in moist and shaded places. It is very easy to grow and multiply and shows attractive metallic blue-green color in the shade and faded in sun exposed places; very suitable for ornamental plant.

Locality: Wonosobo (Wonosobo-city)

Distribution: China (common), NE-India, Japan, Vietnam. Introduced to India (West Bengal), Indonesia (Java), Paraguay, Taiwan, USA (Georgia, Louisiana, Florida).

Specimens observed: ADS 286, ADS 454, ADS 455.

Note: The discovery of $S$. uncinata in the capital district of Wonosobo is a new record for Central Java. The only $S$. uncinata sheets of Herbarium Bogoriense (BO) is the collection of Junghuhn no. 1239 (det. 1910) which may be obtained from the collections of the Bogor Botanical Gardens. The botanical garden has brought a variety of selaginellas from outside Java and abroad, but almost all the introduced selaginellas have died and have not been naturalized. However, in the upland botanical gardens near it, the Cibodas Botanical Gardens $(1350 \mathrm{~m})$, this species was found either planted or growing wild. The presence of $S$. uncinata in this city is presumably introduced as an ornamental plant from southern China, because the Chinese have lived in the city since hundreds of years ago (Setyawan 2012). Besides, the species of Cibodas Botanic Garden can easily be grown in the Depok city $(100 \mathrm{~m})$, indicating that it is not species specific to highlands (data not shown).

Selaginella willdenovii (Desv. ex Poir.) Baker; Gard.

Chron., 783, 950 (1867) (Figure 3.N)

Basionym: Lycopodium willdenovii Desv. ex Poir., Lamarck, Encycl. Suppl. 3: 540, 552, no. 87. [1813] 1814.

Synonym: Lycopodioides caespitosa (BI.) Kuntze, Rev. Gen. Pl. 1: 826 (1891); Lycopodioides willdenowii (Desv.) Kuntze, Rev. Gen. Pl. 1: 827 (1891); Lycopodium bicolor Hort. ex Warburg, Monsunia, 1: 129 (1900); Lycopodium caespitosum Blume, Enum. Pl. Jav., 2: 270, no. 23 (1830); Lycopodium laevigatum Willd., Sp. Pl. 5: 45, no. 66 (1810); Selaginella altissima Kaulf. ex W. Lauche., Verz. August. Gart. 8 (1856) nomen; Selaginella arborea Hort. ex A. Br., App. Ind. Sem. Hort. Berol. 22 (1857); Selaginella arborea Hort. ex Dippel, Amtl. Ber. Deutsch. Naturf. 39 in Giessen (1864), 145, t. 4, f. 16. 1865; Selaginella arborescens Hort. ex Russow, Mem. Acad. Imp. Petersb. VII, 19: 176 (1873); Selaginella caespitosa (Blume) Spring, Bull. Acad. Brux. 10: 140, no. 33 (1843); Selaginella denuana Alderw., Bull. Jard. Bot. Buit. II, 16: 55 (1914); Selaginella laevigata (Willd.) Spring, Mart. Fl. Bras. 1 (2): 125, no. 13 (1840); Selaginella uncinatum var. arborea Mett., Fil. Hort. Lips. 124 (1856); Selaginella willclenovii var. punctulata Alderw., Mal. Ferns \& Fern Allies, Suppl. I, Corn: 40 (1917); Selaginella willdenovii f. typica Alderw., Mal. Ferns \& Fern Allies, Suppl. I, Corr.: 40 (1917); Selaginella willdenovii var. caesia-arborea (Hort.) Hieron. ex R. Bonap. Notes Pterid., 2: (1915).

Common name: Vine spike-moss 
Perennial herb, very stout, pliant, viselike or shrub like, creeping, climbing, scandent, scrambling or hanging, fanshaped frond, 3-4 m long (in experimental garden, regularly pruned, it can be longer); metallic blue-green iridescent leaves in shaded places. Stems are creeping, climbing, hanging, branched from near base upward, 4-5forked branching, not articulate, glabrous with a large, stout main stem, stramineous or reddish, $2.5-8 \mathrm{~mm}$ diam., $50-80 \mathrm{~cm}$ wide (including frond leaves). Rhizophores borne on upper side or underside of stems throughout stem length, mostly at stem base or stem near to the ground, with some spine like protuberances at base (as well as at axes of stems), more rarely in the climbing or hanging ones, 3-4 $\mathrm{mm}$ diam. Leaves are conspicuously dimorphic, delicate, papery, iridescent, green to metallic blue-green, single vein, arranged in 4 lanes (2 lateral, 2 median), but somewhat spirally in stem with monomorphic leaves; lateral leaves distant or approximate, ovate to oblongfalcate, obviously larger than those on branches, asymmetrical, 3-5 $\mathrm{mm}$ long, 2-3 $\mathrm{mm}$ wide (leaves on tertiary stems much smaller); basiscopic base rounded with small auricle, acroscopic base overlapping stem with whitish, long, downward-curving auricle; apex acute, rounded or obtuse, base asymmetrically rounded, margins conspicuously transparent (whitish and shiny when dry), entire, single vein, flattened; median leaves falcatelanceolate or oblique-ovate, imbricate to overlapping at leaf apex, asymmetrical, those on main stems obviously larger than those on branches, 2-3 mm long, 1-2 mm wide, apex acute, acuminate or obtuse, base auriculate, outer auricle larger than inner, margins transparent, entire, single vein; axillary leaves present at branch forks, inserted at the dorsal side of the stem, obviously larger than those on branches, oblong, elliptic to broadly ovate, more equally sided,3-4 mm long, 2-3 mm wide, single vein, apex acute, base rounded to subcordate, biauriculate, margin serrulate. Strobilus solitary, terminal, compact, tetragonal, up to ca. 0.5-4 cm long; sporophylls monomorphic.

Locality: Temanggung (Temanggung-city)

Habitat and ecology: Shady slope of gorge among brushwood. Locally not rare. $450 \mathrm{~m}$. asl. (from herbarium label of JA Lorzing 2510, March 1912 (BO!).

Distribution: Australia (Queensland), Cambodia, India (Arunachal Pradesh, Assam, Himalayas), Indonesia, Laos, Malaysia (Peninsular), Myanmar, Philippines, Singapore, Thailand, Vietnam. In Indonesia: Java, Lesser Sunda Isl., Sumatra. Introduced to: Brazil, Colombia, Jamaica, Panama, Puerto Rico, USA (Florida).

Specimens observed: ADS 460, JA Lorzing 2510 (BO!)

Note: The first published specific epithet is willdenovii, an error spelling, now, the accepted specific epithet is willdenowii, the full accepted name is Selaginella willdenowii (Desv. ex Poir.) Baker (ITIS 2016). Intensive field studies are currently unable to confirm the presence of $S$. willdenowii in the study site, although the herbarium records stated that the species is "locally not rare" in 1912. Extensive research across Java and observations of herbarium collections of $\mathrm{BO}$ indicate that the species is widely grown in the western part of Java, especially in the highlands of Bandung to Ujung Kulon (ADS, 2007-2013, pers. obs.). Environmental changes may have led to the scarcity of this species in Central Java. This species had also found on the southern slope of Mount Slamet, Central Java (ARS 103, 17 March 2004 (BO!). Transplant experiments in Wonosobo indicate that this species can grow well in the garden experiment, and showed bluegreen iridescent that attractive as an ornamental plant, but during the five years of the experiment, there was no naturalized in the wild. The iridescence is caused by the effect of thin film interference filters in the leaf epidermis. The convex epidermal cells may focus light into a single, distal, large chloroplast, possibly adaptations for the improvement of photosynthetic efficiency at the forest floor level (Lee 1977).

\section{ACKNOWLEDGEMENTS}

We thank the head and staff of Herbarium Bogoriense (BO), RCB-IIS, Cibinong-Bogor, Indonesia for facilitated the herbarium materials, Dr. Rogier de Kok of Kew Herbarium for translating the Dutch label, Prof. W.L. Chiou and Dr. Ho-Ming Chang of Taiwan Forestry Research Institute and Dr. Tatik Chikmawati of Bogor Agricultural University for kindly communicating taxonomical characteristics. This research was partly funded by Directorate General of Higher Education, Ministry of Education and Culture, Republic of Indonesia (025/SP2H/KPM/DIT.LITABMAS/V/2013).

\section{REFERENCES}

Alderwereld van Rosenburgh CRWK van. 1915a. Malayan fern allies. Department of Agriculture, Industry, and Commerce. Batavia.

Alderwereld van Rosenburgh CRWK van. 1915b. New or interesting Malay ferns 7. Bull Jard Bot Buitenzorg 2 (20): 1-28.

Alderwereld van Rosenburgh CRWK van. 1916. New or interesting Malay ferns 8. Bull Jard Bot Buitenzorg 2 (23): 1-27.

Alderwereld van Rosenburgh CRWK van. 1917. New or interesting Malay ferns 9. Bull Jard Bot Buitenzorg 2 (24): 1-8.

Alderwereld van Rosenburgh CRWK van. 1918. New or interesting Malay ferns 10. Bull Jard Bot Buitenzorg 2 (28): 1-66.

Alderwereld van Rosenburgh CRWK van. 1920. New or interesting Malay ferns 11. Bull Jard Bot Buitenzorg 3 (2): 129-186.

Alderwereld van Rosenburgh CRWK van. 1922. New or interesting Malay ferns 12. Bull Jard Bot Buitenzorg 3 (5): 179-240.

Alston AHG. 1935a. The Selaginella of the Malay Islands: I. Java and the Lesser Sunda Islands. Bull Jard Bot Buitenzorg 3 (13): 432-442.

Alston AHG. 1935b. The Philippines species of Selaginella. Philippines J Sci 58: 359-383.

Alston AHG. 1937. The Selaginella of the Malay Islands: II. Sumatra. Bull Jard Bot Buitenzorg 3 (14): 175-186.

Alston AHG. 1940. The Selaginella of the Malay Islands: III. Celebes and the Moluccas. Bull Jard Bot Buitenzorg 3 (16): 343-350.

Alston, AHG. 1934. The genus Selaginella in the Malay Peninsula. Gard Bull Strait Settl 8: 41-62.

Andrews SB. 1990. Ferns of Queensland. Queensland Department of Primary Industry, Brisbane.

BPS Banjarnegara. 2011. Banjarnegara in Figures 2011. District of Banjarnegara and BPS Banjarnegara, Banjarnegara [Indonesian]

BPS Kebumen 2011. Kebumen in Figures 2011. District of Kebumen and BPS Kebumen, Kebumen [Indonesian]

BPS Purworejo 2011. Purworejo in Figures 2011. District of Purworejo and BPS Purworejo, Purworejo [Indonesian]

BPS Wonosobo 2011. Wonosobo in Figures 2011. District of Wonosobo and BPS Wonosobo, Wonosobo [Indonesian] 
Camus JM. 1997. The genus Selaginella (Selaginellaceae) in Malesia. In Dransfield J (ed). Plant Diversity of Malesia III: 59-69.

Chang HM, Chiou WL, Wang JC. 2012. Flora of Taiwan, Selaginellaceae. Endemic Species Research Institute, Nantou, Taiwan.

Chikmawati T, Setyawan AD, Miftahudin. 2012. Phytochemical Composition of Selaginella spp. from Java Island Indonesia. Makara J Sci 16 (2): 129-133

Hassler M, Swale B. 2002. Checklist of World Ferns. http://homepages.caverock.net.nz/ bj/fern/

ITIS [Integrated Taxonomic Information System]. 2016. Selaginella willdenowii (Desv. ex Poir.) Baker; Taxonomic Serial No.: 505115. http://www.itis.gov/servlet/SingleRpt/SingleRpt?search_topic=TSN\& search value $=505115$

Jermy AC. 1990. Selaginellaceae. In: Kubitzki K, Kramer KU, Green PS (eds.). The Families and Genera of Vascular Plants, 1. Pteridophytes and Gymnosperms. Springer, Berlin.

Junghuhn F. 1855. Kaart van het Eiland Java-Topografische. A.J. Bogaerts Ausgabe, Germany.

Junghuhn F. 1860. Geographisch-Physikalische von Java-Topografische. Justus Perthes, Germany.

Lee DW. 1977. On iridescent plants. Gard Bull Singapore 30: 21-29

Li ZJ, Tan BC. 2005. A review of the species diversity of Selaginella in Fujian Province of China. Acta Phytotaxonomica Sinica 43 (1): 50-59

Oldeman LR., 1975. Agroclimatic map of Java \& Madura. Contr. of Centra. Res. Inst. for Food Crops. Bogor.

Setyawan AD, Sugiyarto, Susilowati A, Widodo. 2015a. Diversity and distribution of Selaginella in the Province of Yogyakarta Special Region. Pros Sem Nas Masy Biodiv Indon 1: 987-992.

Setyawan AD, Sugiyarto, Susilowati A, Widodo. 2015b. Diversity of Selaginella in the karstic region of Sewu Mountains, Southern Java. Pros Sem Nas Masy Biodiv Indon 1: 1318-1323.

Setyawan AD, Sugiyarto, Widiastuti A. 2012. Species diversity of Selaginella in Mount Merapi National Park, the central part of Java. Proc Soc Indon Biodiv Intl Conf 1: 102-106

Setyawan AD, Sugiyarto, Widiastuti A. 2015c. Species diversity of Selaginella in the Dieng Plateau, Central Java. Pros Sem Nas Masy Biodiv Indon 1: 980-986.

Setyawan AD, Sugiyarto. 2015. Diversity of Selaginella in the Bromo Tengger Semeru National Park, East Java. Pros Sem Nas Masy Biodiv Indon 1: 1312-1317

Setyawan AD, Sutarno, Sugiyarto. 2013. Species diversity of Selaginella in Mount Lawu, Java, Indonesia. Biodiversitas 14 (1): 1-9.
Setyawan AD. 2008. Species richness and geographical distribution of Malesian Selaginella. The 8th Seminary and Congress of Indonesian Plant Taxonomy Association ("PTTI"), Cibinong Science Center, Bogor-Indonesia, 21-23 October 2008

Setyawan AD. 2009. Traditionally utilization of Selaginella; field research and literature review. Nusantara Bioscience 1 (3): 146-158.

Setyawan AD. 2011. Natural products from Genus Selaginella (Selaginellaceae). Nusantara Bioscience 3 (1): 44-58.

Setyawan AD. 2012. Altitudinal distribution of Selaginella in the southern part of Central Java, Indonesia. Proc Soc Indon Biodiv Intl Conf 1: 153-157.

Setyawan AD. 2014. A new record of naturalized Selaginella uncinata (Desv.) Spring (Selaginellaceae) from Java, Indonesia. Biodiversitas 15: 261-268.

Setyawan AD. 2015a. Diversity and distribution of Selaginella in the southern West Java based on altitudinal gradient. Pros Sem Nas Masy Biodiv Indon 1: 490-495.

Setyawan AD. 2015b. Diversity of Selaginella in the Province of Banten, Western Java. Pros Sem Nas Masy Biodiv Indon 1: 1810-1815

SRI [Soil Research Institute]. 1960. Exploratory soil map of Java and Madura. Indonesian Ministry of Agriculture, Bogor

Tryon RM, Tryon AF. 1982. Fern and Allied Plants, with Special Reference to Tropical America. Springer, New York.

Tsai JL, Shieh WC. 1994. Selaginellaceae. In: Huang TC (ed) Flora of Taiwan. Vol. 1. 2nd ed. Department of Botany, National Taiwan University, Taipei

van Bemmelen RW. 1949. The geology of Indonesia. Martinus Nijhoff, The Hague.

Winter WP de, Jansen PCM. 2003. Selaginella Pal. Beauv. In: de Winter WP, Amoroso VB (eds). Plant resources of South-East Asia 15 (2) Cryptogams: ferns and fern allies. Backhuys, Leiden.

Wong KM. 1982. Critical observations on Peninsular Malaysian Selaginella. Gard Bull Sing 35 (2): 107-135

Wong KM. 2010. Selaginellaceae. In Parris BS, Kiew R, Chung RKC, Saw LG, Soepadmo E (eds) Flora of Peninsular4 Malaysia Series 1. Ferns and Lycophytes. Malayan Forest Records No. 48. FRIM Kepong, Selangor.

Zhang XC, Nooteboom HP, Kato M. 2013. Selaginellaceae. In: Wu ZY, Raven PH, Hong DY (eds.). Flora of China, Vol. 2-3 (Pteridophytes). Science Press, Beijing \& Missouri Botanical Garden Press, St. Louis. 